\title{
On the connection of Hypergraph Theory with Formal Concept Analysis and Rough Set Theory ${ }^{1}$
}

\author{
Gianpiero Cattaneo* and Giampiero Chiaselotti ${ }^{\circ}$ and Davide Ciucci* and Tommaso \\ Gentile $^{o}$ \\ * Department of Informatics, Systems and Communications, University of Milano-Bicocca, 20126 \\ Milano, Italy \\ ${ }^{\circ}$ Department of Mathematics and Informatics, University of Calabria, Via Pietro Bucci, Cubo 30B, \\ 87036 Arcavacata di Rende (CS), Italy.
}

\begin{abstract}
We present a unique framework for connecting different topics: hypergraphs from one side and Formal Concept Analysis and Rough Set Theory from the other. This is done through the formal equivalence among Boolean information tables, formal contexts and hypergraphs. Links with generic (i.e., not Boolean) information tables are established, through so-called nominal scaling. The particular case of $k$-uniform complete hypergraphs will then be studied. In this framework, we are able to solve typical problems of Rough Set Theory and Formal Concept Analysis using combinatorial techniques. More in detail, we will give a formula to compute the degree of dependency and the partial implication between two sets of attributes, compute the set of reducts and define the structure of the partitions generated by all the definable indiscernibility relations.
\end{abstract}

Keywords: Rough sets, Formal Concept Analysis, Hypergraphs

\section{Introduction}

Hypergraph theory, Formal Context Analysis and Rough Set Theory are three welldeveloped fields of study.

Hypergraph theory is a generalization of graph theory (see [4, 5]) where edges, called hyperedges, can have an arbitrary number of vertices. Classically, the typical problems studied in hypergraph theory concern combinatorial questions (see $[5,8]$ ) and optimization questions (see [20]).

Formal Context Analysis (briefly FCA) deals with structures called Formal Contexts [17], that describe objects in terms of the properties they possess. A formal concept is an objects-properties pair $(O, P)$ such that the objects in $O$ are all and the only to satisfy all the properties in $P$. Starting then from the definition of formal concept, the

\footnotetext{
${ }^{1}$ Appeared in Information Sciences, 330, 342-357 (2016). DOI: 10.1016/j.ins.2015.09.054

Email address:

cattang@live.it,giampiero.chiaselotti@unical.it,ciucci@disco.unimb.it,gentile@mat.unical.it (Gianpiero Cattaneo* and Giampiero Chiaselotti ${ }^{\circ}$ and Davide Ciucci* and Tommaso Gentile ${ }^{\circ}$ )

Preprint submitted to Information Sciences

January 1, 2016
} 
FCA theory developed as a very powerful theoretical methodology useful to approach problems in data mining, machine learning and related fields (see [21]).

Finally, an Information System (or information table) collects the values that a set of objects have on some attributes. Objects are then partitioned according to an equivalence relation in classes of objects with equal values for all attributes. The pair $(U, R)$ made of the objects and the equivalence relation is called approximation space, and it can be analyzed by using the methods derived from Rough Set Theory (RST) (see [26, 27]).

In particular, an Information System is called Boolean if we fix the set of attribute values equal to $\{0,1\}$ and it is easily proved to be equivalent to a formal context. See for instance [36], [37] and [38], where a unifying approach between FCA theory and Boolean Information System theory has been outlined. Let us notice that several authors studied the link between RST and FCA and mixed the two theories in several ways, see for instance $[18,19,25,32,33]$.

On the other hand, in several other papers (see [14], [15], [30], [31]) specific hypergraphs associated with some particular type of formal contexts and Boolean information tables were constructed. For instance, Stell applies rough set and formal concept ideas to hypergraphs: in [30] rough hypergraph theory is introduced and, similarly, in [31] FCA are generalized using a relation on hypergraphs instead of sets. In [14] a hypergraph is considered as a basic model for granular structures. Results in [15] directly put in relation a formal context with a hypergraph but differently as we do (see Remark 3.9).

In this paper, we survey some results concerning the relationship among formal contexts, Boolean information tables and hypergraphs. Moreover, through a scaling procedure we see that information tables (not necessarily Boolean) are in relation with a sub-class of hypergraphs. Several consequences are then derived from this unified framework. First of all, in the next example we show how some questions concerning a typical problem derived from an information table can be naturally interpreted as a combinatorial problem in hypergraph theory.

Example 1.1. Let us consider an information table with 100 distinct attributes Att $=$ $\left\{a_{1}, \ldots, a_{100}\right\}$ and suppose that we can uniquely characterize any object by exactly 7 attributes (not necessarily the same for all objects). For instance, the sequence of attributes

$$
a_{1} a_{3} a_{4} a_{15} a_{23} a_{71} a_{98}
$$

uniquely determine a specific object of our universe and, on the other hand, each object of our universe is uniquely determined by a "code" of length 7 of distinct attributes selected from $a_{1}, \ldots, a_{100}$. In this case, we can represent such a situation as a new Boolean Information System, where the attributes are $A t t:=\left\{a_{1}, \ldots, a_{100}\right\}$, the objects are all the 7 -subsets $u, v, \ldots$ of $A t t$ and the value of an object $u$ on an attribute $a$ is equal to 1 if $a \in u$ and equal to 0 otherwise. We choose now a particular subset $A$ of Att, such that $A$ has at most seven elements. For example $A=\left\{a_{1}, a_{2}, a_{50}, a_{51}, a_{97}\right\}$. Let us suppose that a user wants to know the following information:

1. How many distinct equivalence classes of items with respect to attributes $A$ are there?

2. Which are the items having as part of their code the sub-string $a_{2} a_{50} a_{97}$, but none of the symbols $a_{1}, a_{51}$ ? 
The previous can be considered two very practical requests for a user, and they can be understood more effectively after being framed in Information System theory, by using combinatorial techniques (see Remark 4.5 and Proposition 4.6). Let us note that, in terms of Information Systems, the request (2) is equivalent to determine all the objects in our universe that are in a specific $A$-indiscernibility class, namely those with attributes $a_{2} a_{50} a_{97}$ equal to 1 and attributes $a_{1} a_{51}$ equal to 0 in the original information table.

The previous example shows that it can be useful to define and to study some combinatorial properties of Information Systems where we have a fixed set of attributes $a_{1}, a_{2}, \ldots$ and where each object of the universe is uniquely determined by a choice of distinct attributes. Hence, each object $u$ can be identified with some subset of $a_{1}, a_{2}, \ldots$, and this situation can be represented by a hypergraph, where attributes are the vertices and objects are the hyperedges. Mathematical literature (see [4], [5], [8], [20]) provides several examples of hypergraph families. A classical example of deeply studied hypergraph family are (n,k) uniform complete hypergraphs $\left\{\left(\begin{array}{l}\hat{n} \\ k\end{array}\right): n \geq k \geq 0\right\}$, where $\left(\begin{array}{l}\hat{n} \\ k\end{array}\right)$ is the set of all subsets with $k$ elements of the $n$-set $\hat{n}:=\{1, \ldots, n\}$. This family of hypergraphs is exactly the one needed to answer questions (1) and (2) above.

Moreover, as another example of the possibility given by the connection among information tables, formal contexts and hypergraphs, we will study the notion of attribute dependency with the help of $k$-uniform complete hypergraphs. In particular we will be able to compute the RST-dependency degree of a set of attributes $B$ with respect to a set of attributes $A$ and the FCA-precision of a partial implication [23].

The paper is organized as follows. In Section 2, we give the basic definitions of the three involved theories: rough set theory (in particular, information tables, approximations and attribute dependence), formal context analysis, hypergraphs. The equivalence of the three involved structures to represent data is then given in Section 3. In Section 4 we study in detail our "hypergraph model" $\left(\begin{array}{c}\hat{n} \\ k\end{array}\right)$ and we apply on this specific model all the general theoretical tools introduced in the previous sections. In Section 5 we define the notion of granular partition lattice for a hypergraph and we determine this lattice for the hypergraph $\left(\begin{array}{l}\hat{n} \\ k\end{array}\right)$. Moreover, we establish an isomorphism between the granular partition lattice and another lattice derived from pattern structures, as introduced in [16]. Finally, in Section 6 we draw some conclusions and outline future works.

\section{Basic Notions}

In this section, the preliminary notions of Rough Set Theory, Formal Concept Analysis and Hypergraphs are introduced.

\subsection{Rough Set Theory}

An Information System is a structure $\mathcal{J}=\langle U, A t t, V, F\rangle$, where $U$ (called universe set) is a non empty set of elements called objects, Att (called attribute set) is a non empty set of elements called attributes, $V$ (called value set) such that $V=\bigcup_{a \in A t t} V_{a}$ where $V_{a}$ is the set of values that attribute $a$ can assume and each $V_{a}$ is non empty and $F: U \times A t t \rightarrow V$ (called information map) is a mapping from the direct product $U \times A t t$ into the value set $V$. 
From now on, we assume that both the object set $U$ and the attribute set $A$ are finite and we also identify the attribute $a \in A t t$ with the map $f_{a}: U \rightarrow V_{a}$ defined by $f_{a}(u):=F(u, a)$.

Usually an Information System is represented by a matrix $T[\mathcal{J}]$, whose rows are indexed with the objects $u_{1}, u_{2}, \ldots$ and whose columns are indexed with the attributes $a_{1}, a_{2}, a_{3}, \ldots$ By definition, the table $T[\mathcal{J}]$ contains in the place $(n, m)$ the value $F\left(u_{n}, a_{m}\right)$. If $V=\{0,1\}$ we say that $\mathcal{J}$ is a Boolean Information System.

If $A \subseteq A t t$, it is usual to consider the binary relation $I_{A}$ on the universe set $U$ defined as follows: if $x, y \in U$ then

$$
x I_{A} y \Longleftrightarrow \forall a \in A, f_{a}(x)=f_{a}(y) .
$$

The binary relation $I_{A}$ is an equivalence relation on $U$ and it is called indiscernibility relation generated by $A$ (briefly $A$-indiscernibility relation). If $x \in U$, we denote by $[x]_{A}$ the equivalence class generated by $x$ with respect to $I_{A}$ and we also set $\pi_{A}(\mathcal{J}):=\left\{[x]_{A}\right.$ : $x \in U\}$ the partition of $U$ induced by $I_{A}$ (when $\mathcal{J}$ is clear from the context we write simply $\pi_{A}$ instead of $\left.\pi_{A}(\mathcal{J})\right)$. Any set $[x]_{A}$ is called an A-elementary set of $U$.

Definition 2.1. Let $\mathcal{J}=\langle U, A t t, V, F\rangle$ be an Information System and $A \subseteq$ Att. A subset $E \in U$ is called $A$-exact (or crisp) if and only if $E$ is either the empty set or an $A$ elementary set or the set-theoretic union of some A-elementary sets. We will denote by $\mathbb{C O}_{A}(\mathcal{J})$ the set of all the $A$-exact subsets of the universe set $U$ of the Information System J.

If $Y$ is a subset of the universe set $U$ we recall the concepts of lower and upper approximation of $Y$ with respect to $A \subseteq A t t$. The A-lower approximation of $Y$ is the following subset of the universe $U$ :

$$
\mathbf{l}_{A}(Y):=\left\{x \in U:[x]_{A} \subseteq Y\right\}
$$

On the other hand, the A-upper approximation of $Y$ is defined as:

$$
\mathbf{u}_{A}(Y):=\left\{x \in U:[x]_{A} \cap Y \neq \emptyset\right\} .
$$

Let us note that

$$
\mathbf{l}_{A}(Y)=\bigcup\left\{[x]_{A}:[x]_{A} \subseteq Y\right\}
$$

and

$$
\mathbf{u}_{A}(Y)=\bigcup\left\{[x]_{A}:[x]_{A} \cap Y \neq \emptyset\right\} .
$$

Therefore both $\mathbf{l}_{A}(Y)$ and $\mathbf{u}_{A}(Y)$ are $A$-exact subsets of $U$, for each subset $Y \subseteq U$. Trivially, for any subset $Y$ of $U$ one has that $\mathbf{l}_{A}(Y) \subseteq Y \subseteq \mathbf{u}_{A}(Y)$. As a consequence the pair $r_{A}(Y):=\left(\mathbf{l}_{A}(Y), \mathbf{u}_{A}(Y)\right)$ is called the rough set of $Y$ relatively to $A$. Moreover, it is easy to see that for each $Y \subseteq U, Y \in \mathbb{C O}_{A}(\mathcal{J})$ if and only if $\mathbf{l}_{A}(Y)=\mathbf{u}_{A}(Y)$.

Remark 2.2. It is well known that the A-exact set family $\mathbb{C} \mathbb{O}_{A}(\mathcal{J})$ has very nice properties. At first it defines an Alexandroff topology on the universe set $U$, where each open set is also closed and vice versa (this justifies the name $\mathbb{C} \mathbb{O}$, which stands for Closed 
and Open $)^{2}$. Moreover, $\mathbb{C O}_{A}(\mathcal{J})$ is a field of sets on $U$ which is complete, i.e., a family of subsets of $U$ which contains both the empty set and the total set $U$, and is closed under set-theoretic intersections $\cap$ and unions $\cup$ and the set theoretic operation of complementation ${ }^{c}$. Hence $\mathbb{C O}_{A}(\mathcal{J})$ gives rise to a Boolean sub-algebra of the Boolean algebra $\left\langle\mathcal{P}(U), \subseteq, \cap, \cup,{ }^{c}, \emptyset, U\right\rangle$. More precisely, if $\pi_{A}(\mathcal{J})$ contains exactly s elements (i.e. equivalence classes), then $\mathbb{C O}_{A}(\mathcal{J})$ is a Boolean algebra isomorphic to $\left\langle\mathcal{P}(\hat{s}), \subseteq, \cap, \cup,{ }^{c}, \emptyset, \hat{s}\right\rangle$, where $\hat{s}:=\{1,2, \ldots, s\}$.

If $\mathcal{J}=\langle U, A t t, V, F\rangle$ is an Information System and we fix a subset $B \subseteq A t t$ and a subset $Z \subseteq U$, the rough membership function $\mu_{Z}^{B}: U \rightarrow[0,1]$ is defined as follows (see $[26])$ :

for all $u \in U$.

$$
\mu_{Z}^{B}(u):=\frac{\left|[u]_{B} \cap Z\right|}{\left|[u]_{B}\right|}
$$

Definition 2.3. Let $\mathcal{J}=\langle U, A t t, V, F\rangle$ and $\mathcal{J}^{\prime}=\left\langle U^{\prime}, A t t^{\prime}, V^{\prime}, F^{\prime}\right\rangle$ be two Information Systems. We say that $\mathcal{J}$ and $\mathcal{J}^{\prime}$ are isomorphic if there exist three bijective maps $\alpha: U \rightarrow$ $U^{\prime}, \beta: A t t \rightarrow A t t^{\prime}$ and $\gamma: V \rightarrow V^{\prime}$ such that $F^{\prime}(\alpha(u), \beta(a))=\gamma(F(u, a))$ for all $u \in U$ and $a \in$ Att. In this case we write $\mathrm{J} \cong \mathrm{J}^{\prime}$.

Obviously, if $\mathcal{J}$ and $\mathcal{J}^{\prime}$ are isomorphic, then $V=V^{\prime}$ and $T[\mathcal{J}]=T\left[\mathcal{J}^{\prime}\right]$.

We recall now the notion of attribute dependency in rough set theory. Let $\mathcal{J}=$ $\langle U, A t t, V, F\rangle$ be an Information System and let $A, B$ be two subsets of Att such that $\pi_{B}(\mathcal{J})=\left\{Q_{1}, \ldots, Q_{N}\right\}$. The subset $\operatorname{Pos}_{A}(B):=\bigcup_{i=1}^{N} \mathbf{l}_{A}\left(Q_{i}\right)$ is called $A$-positive region of $B$. The $A$-degree dependency of $B$ (see [26]) is the number

$$
\gamma_{A}(B):=\frac{\left|\operatorname{Pos}_{A}(B)\right|}{|U|}
$$

This number measures a type of $A$-degree exactness of the attribute subset $B$ with respect to the attribute subset $A$, that is, how many objects of the $B$ equivalence classes can be classified with certainty using attributes in $A$. Let us note that, since $Q_{1}, \ldots, Q_{N}$ are pairwise-disjoint, we have $\left|\operatorname{Pos}_{A}(B)\right|=\sum_{i=1}^{N}\left|\mathbf{l}_{A}\left(Q_{i}\right)\right|$ and so the above (3) assumes the form

$$
\gamma_{A}(B):=\frac{\sum_{i=1}^{N}\left|\mathbf{l}_{A}\left(Q_{i}\right)\right|}{|U|}
$$

Finally, a reduct of $\mathcal{J}$ is an attribute subset that fully characterize the knowledge in $\mathcal{J}$ and is minimal with respect to this property. In other terms, no attribute can be removed from this subset without causing a loss of knowledge. Formally,

Definition 2.4. Let $\mathcal{J}=\langle U, A t t, V a l, F\rangle$ be an Information System. An attribute $c \in$ Att is said indispensable if $\pi_{A t t} \neq \pi_{A t t \backslash\{c\}}$. The subset of all indispensable attributes of Att is called core of $\mathcal{J}$ and it is denoted by $C O R E(\mathcal{J}) . C$ is said a reduct of $\mathcal{J}$ if:

(i) $\pi_{\text {Att }}=\pi_{C}$;

(ii) $\pi_{A t t} \neq \pi_{C \backslash\{c\}}$ for all $c \in C$.

We denote by $R E D(\mathcal{J})$ the family of all reducts of $\mathcal{J}$.

\footnotetext{
${ }^{2}$ See $[1,2]$, and $[10]$ for a discussion of this topology and its Tarski and Kuratowski weakening in the context of rough set theory.
} 
The following result connects core and reducts of $\mathcal{J}$.

Proposition 2.5. [26] $C O R E(\mathcal{J}):=\bigcap\{C: C \in R E D(\mathcal{J})\}$.

\subsection{Formal Concept Analysis}

We recall now the notion of a formal context.

Definition 2.6. A Formal Context is a triple $\mathbb{K}=(G, M, \mathcal{R})$, where $G$ and $M$ are sets and $\mathcal{R} \subseteq G \times M$ is a binary relation. The elements of $G$ and $M$ are called objects and attributes, respectively. We write $g \mathcal{R} m$ instead of $(g, m) \in \mathcal{R}$. If $O \subseteq G$ and $Q \subseteq M$, we set

$$
O^{\uparrow}:=\{m \in M:(\forall g \in O) g \mathcal{R} m\} \subseteq M
$$

and

$$
Q^{\downarrow}:=\{g \in G:(\forall m \in Q) g \mathcal{R} m\} \subseteq G .
$$

In this way the following two mappings are defined: ${ }^{\uparrow}: \mathcal{P}(G) \rightarrow \mathcal{P}(M), O \rightarrow O^{\uparrow}$ and $\downarrow: \mathcal{P}(M) \rightarrow \mathcal{P}(G), Q \rightarrow Q^{\downarrow}$.

Definition 2.7. A formal concept of the formal context $\mathbb{K}=(G, M, \mathcal{R})$ is a pair $(O, Q)$, where $O \subseteq G, Q \subseteq M, O^{\uparrow}=Q$ and $Q^{\downarrow}=O$. If $(O, Q)$ is a formal concept, $O$ is called the extent of $(O, Q)$ and $Q$ is called the intent of $(O, Q)$. We denote by $\mathfrak{B}(\mathbb{K})$ the set of all the formal concepts of the formal context $\mathbb{K}$.

By composition of the above maps we obtain the two operators * : $\mathcal{P}(G) \rightarrow \mathcal{P}(G), O \rightarrow$ $O^{\uparrow \downarrow}$ and ${ }^{\diamond}: \mathcal{P}(M) \rightarrow \mathcal{P}(M), Q \rightarrow Q^{\downarrow \uparrow}$.

Remark 2.8. These are both closure operators [6, Theorem 19, p.123]. Indeed, and discussing the first case since the second can be developed in a similar way, the following hold: (C1) $\forall O \in \mathcal{P}(G), O \subseteq O^{*}$ and (C2) $O^{*}=O^{* *}$, finally (C3) $\forall O_{1}, O_{2} \in \mathcal{P}(G)$, $O_{1}^{*} \cup O_{2}^{*} \subseteq\left(O_{1} \cup O_{2}\right)^{*}$. In general $\emptyset \subseteq \emptyset^{*}$, but if one introduces the new mapping $u: \mathcal{P}(G) \rightarrow \mathcal{P}(G)$ defined for every subset $O \in \mathcal{P}(G) \backslash\{\emptyset\}$ as $u(O)=O^{*}$ and setting $u(\emptyset)=\emptyset$, then in this case we have a Tarski closure operator since: $(C 0) u(\emptyset)=\emptyset$, (C1) $\forall A \in \mathcal{P}(G), A \subseteq u(A)$ and (C2) $u(A)=u\left(u(A)\right.$ ), finally (C3) $\forall A_{1}, A_{2} \in \mathcal{P}(G)$, $u\left(A_{1}\right) \cup u\left(A_{2}\right) \subseteq u\left(A_{1} \cup A_{2}\right)$.

For any Tarski closure operation it is possible to introduce the corresponding family of closed subsets $\mathcal{C}(G):=\{C \in \mathcal{P}(G): C=u(C)\}$ and $\mathcal{C}(M):=\{K \in \mathcal{P}(M): K=w(K)\}$, if we denote with $w$ the analogous of $u$ in the case of $M$, which are both nonempty since they contain the empty set and the whole space. Moreover, they are closed with respect to arbitrary set-theoretic intersections, i.e., they are pre-topologies but not topologies since equality in (T4) does not hold.

This should be compared with the rough set case as discussed in remark 2.2.

If $\left(O_{1}, Q_{1}\right)$ and $\left(O_{2}, Q_{2}\right)$ are two formal concepts in $\mathfrak{B}(\mathbb{K})$, it is usual to consider the relation $\left(O_{1}, Q_{1}\right) \sqsubseteq\left(O_{2}, Q_{2}\right)$ if and only if $O_{1} \subseteq O_{2}$ (which is equivalent to $Q_{1} \supseteq Q_{2}$ ). Then $\sqsubseteq$ is a partial order on $\mathfrak{B}(\mathbb{K})$ and $(\mathfrak{B}(\mathbb{K}), \sqsubseteq)$ is a complete lattice, called concept lattice of the formal context $\mathbb{K}$, whose meet and join operations on an arbitrary family 
of formal concepts $\left\{\left(O_{\alpha}, Q_{\alpha}\right): \alpha \in A\right\}$ are the following:

$$
\begin{aligned}
& \bigwedge_{\alpha \in A}\left(O_{\alpha}, Q_{\alpha}\right)=\left(\bigcap_{\alpha \in A} O_{\alpha},\left(\bigcup_{\alpha \in A} Q_{\alpha}\right)^{\diamond}\right) \\
& \bigvee_{\alpha \in A}\left(O_{\alpha}, Q_{\alpha}\right)=\left(\left(\bigcup_{\alpha \in A} O_{\alpha}\right)^{*}, \bigcap_{\alpha \in A} Q_{\alpha}\right)
\end{aligned}
$$

The following is straightforward.

Proposition 2.9. Let $\mathbb{K}=(G, M, \mathcal{R})$ be a formal context. Then

i) If $(O, Q)$ is a formal concept from $\mathbb{K}$, then $O$ is a $G$-closed and $Q$ a $M$-closed set. Notice that the inverse in general does not hold.

ii) Let $\left(O, O^{\uparrow}\right)$ be a pair of subsets from $\mathcal{P}(G) \times \mathcal{P}(M)$, then this pair is a formal concept iff $O$ is $G$-closed, i.e., $O=O^{*}=O^{\uparrow \downarrow}$.

iii) Let $\left(Q^{\downarrow}, Q\right)$ be a pair of subsets from $\mathcal{P}(G) \times \mathcal{P}(M)$, then this pair is a formal concept iff $Q$ is $M$-closed, i.e., $Q=Q^{\diamond}=Q^{\downarrow \uparrow}$.

The dependency among attributes is studied in FCA under the name implication [17]. An implication $A \rightarrow B$ between sets of attributes $A$ and $B$ holds if $A^{\downarrow} \subseteq B^{\downarrow}$, that is if all the objects sharing properties in $A$ also have properties in $B$. Thus, an implication in this sense implies an exact dependence in the rough set case, i.e., $\gamma_{A}(B)=$ 1. General association rules [28] have been investigated in [23] under the name of Partial implications.

Definition 2.10. $A$ partial implication $A \rightarrow^{p} B$ is a triple $(A, B, p)$ where $A, B \subseteq M$ are sets of attributes and $p:=\delta_{A}(B)$ is the precision or confidence of the implication and it is defined as

$$
\delta_{A}(B)=\frac{\left|(A \cup B)^{\downarrow}\right|}{\left|A^{\downarrow}\right|}
$$

if $A^{\downarrow} \neq \emptyset$, and $\delta_{A}(B)=1$ otherwise.

\subsection{On the Relationship between Formal Contexts and Information Tables}

It is immediate to observe that any formal context can be uniquely associated to a Boolean information table and vice versa. In fact, if $\mathbb{K}=(G, M, \mathcal{R})$ is a formal context, in order to see $\mathbb{K}$ as a Boolean information table $\mathcal{J}=\langle U, A t t,\{0,1\}, F\rangle$ it is sufficient to set $U:=G$, Att $:=M$, and $F(g, m)=1$ if $g \mathcal{R} m, F(g, m)=0$ otherwise. On the other hand, if the Boolean information table $\mathcal{J}=\langle U, A t t,\{0,1\}, F\rangle$ is given, we can identify $\mathcal{J}$ with the formal context $\mathbb{K}:=(G, M, \mathcal{R})$, where $G:=U, M:=A t t$ and $g \mathcal{R} m$ if and only if $F(g, m)=1$.

Moreover, many-valued contexts (for a formal definition see [17]) are an extension of formal contexts such that an attribute can assume different values, not just $\{0,1\}$. Clearly, many-valued contexts are the same as information tables.

In FCA an important topic is how to transform a many-valued context into a formal context (otherwise said how to transform an information table into a Boolean information table). This operation is named scaling and several ways to perform it are known [17]. For the scope of the present paper, we recall one of the elementary scaling techniques, known as nominal scaling. Let us show how it works on an example. 


\begin{tabular}{|c|cccc|}
\hline Patient & Headache & Muscle-pain & Temperature & Flu \\
\hline$p_{1}$ & no & yes & high & yes \\
$p_{2}$ & yes & no & high & no \\
$p_{3}$ & yes & yes & very high & yes \\
$p_{4}$ & no & yes & normal & no \\
$p_{5}$ & yes & no & high & no \\
$p_{6}$ & yes & yes & normal & yes \\
\hline
\end{tabular}

Table 1: Example of information table.

Example 2.11. Let us consider the information table based on the universe $U=$ $\left\{p_{1}, p_{2}, p_{3}, p_{4}, p_{5}, p_{6}\right\}$ of six patients described by the Table 1 .

This information table can be represented as a formal context through a nominal scaling. The idea is to consider as attributes of the associated formal context the set $M:=$ $\bigcup_{a \in A t t}\left\{\left(a, f_{a}(u)\right) \in\right.$ Att $\left.\times V_{a}: u \in U\right\}$, or equivalently, by setting $M_{a}:=\left\{\left(a, f_{a}(u)\right)\right.$ : $u \in U\}$ for every $a \in A t t$ and $M=\bigcup_{a \in A t t} M_{a}$. Hence, in the formal context $\mathbb{K}=$ $\left(U, \bigcup_{a \in A t t} M_{a}, \mathcal{R}\right)$ the binary relation $\mathcal{R} \subseteq U \times \bigcup_{a \in A t t} M_{a}$ is defined according to the following definition:

$$
\text { Let } x \in U, m \in M \text {. Then }(x, m) \in \mathcal{R} \quad \text { iff } \quad \exists a \in A t t: m=\left(a, f_{a}(x)\right)
$$

So, in our example, we obtain the formal context in Table 5.1, where $M=\{\mathrm{H}$-yes, H-no, M-yes, M-no, T-n, T-h, T-vh, F-y, F-n\}, where $H$ is an abbreviation for Headache, $\mathrm{M}$ for Muscle-pain and so on.

\begin{tabular}{|c||c|c||c|c||c|c|c||c|c|}
\hline Patient & H-yes & H-no & M-yes & M-no & T-n & T-h & T-vh & F-y & F-n \\
\hline$p_{1}$ & 0 & 1 & 1 & 0 & 0 & 1 & 0 & 1 & 0 \\
$p_{2}$ & 1 & 0 & 0 & 1 & 0 & 1 & 0 & 0 & 1 \\
$p_{3}$ & 1 & 0 & 1 & 0 & 0 & 0 & 1 & 1 & 0 \\
$p_{4}$ & 0 & 1 & 1 & 0 & 1 & 0 & 0 & 0 & 1 \\
$p_{5}$ & 1 & 0 & 0 & 1 & 0 & 1 & 0 & 0 & 1 \\
$p_{6}$ & 1 & 0 & 1 & 0 & 1 & 0 & 0 & 1 & 0 \\
\hline
\end{tabular}

Table 2: Formal context induced from Table 1.

Where in each row for any set of values $M_{a}$ and object $x$ there is just one 1 in the table, and so the number of $1 \mathrm{~s}$ is the same for every row.

Clearly by the above procedure, starting from an information system $\mathcal{J}=\langle U, A t t, V, F\rangle$ one can construct the Information table $T[\mathcal{J}]$, and then by means of nominal scaling we obtain the formal context $\mathbb{K}=\left(U, \bigcup_{a \in A t t} M_{a}, \mathcal{R}\right)$. If we represent this formal context $\mathbb{K}$ as the Boolean information table $\mathcal{J}^{\prime}=<U, \bigcup_{a \in A t t} M_{a},\{0,1\}, F^{\prime}>$, we have that two objects $x, y \in U$ are indiscernible in the original system $\mathcal{J}$ iff they are indiscernible in the derived one $\mathfrak{J}^{\prime}$. More precisely the following proposition holds. 
Proposition 2.12. Let $\mathcal{J}=\langle U, A t t, V, F\rangle$ be an Information System with Information Table $T[\mathcal{J}]$ and $\mathcal{J}^{\prime}=\left\langle U, \bigcup_{a \in A t t} M_{a},\{0,1\}, F^{\prime}\right\rangle$ the corresponding Boolean transformation of $\mathcal{J}$ through nominal scaling with information table $T\left[\mathcal{J}^{\prime}\right]$. Then,

1. Let $A \subseteq$ Att and $M_{A}=\bigcup_{a \in A} M_{a}$. Let $I_{A} \subseteq U \times U$ be the indiscernibility relation induced on $U$ by the set of attributes $A$ from the system $\mathcal{J}$ and let $I_{M_{A}}^{\prime}$ be the indiscernibility relation induced on $U$ by the set of attributes $M_{A}$ from the system $\mathrm{J}^{\prime}$, then $\forall x, y \in U, x I_{A} y$ iff $x I_{M_{A}}^{\prime} y$.

2. Each row of $T\left[\mathcal{J}^{\prime}\right]$ has exactly $\mid$ Att $\mid$ values equal to 1.

Proof. It easily follows from the scaling procedure.

The second result of the above proposition will be very important in the following since it will enable us to connect general (that is non necessarily Boolean) information tables with hypergraphs. We also remark that despite the formal result that Boolean information tables obtained by a scaling procedure are formal contexts, the two concepts are conceptually different. Indeed, as outlined above, in the case of the information table, each property represents a value with the consequence that properties are not independent. This will reflect also in the equivalence results given in section 3 and summarized in Figure 1.

\subsection{Hypergraph Theory}

Finally, let us define hypergraphs. If $k$ is a non-negative integer and $Y$ is a finite set such that $|Y| \geq k$, we denote by $\left(\begin{array}{c}Y \\ k\end{array}\right)$ the family of all the $k$-subsets of $Y$ (i.e. the subsets of $Y$ with exactly $k$ elements).

Remark 2.13. In what follows we use the term family of subsets of a set $X$. In this case we admit the possibility that such a family can contain two equal elements. This terminology is frequently adopted in mathematical literature and it is useful to distinguish the notions of family and set. Usually, the elements of a set (or a subset) are all distinct, whereas the elements of a family can also be repeated. In this paper we will stick to this terminological distinction.

Definition 2.14. A hypergraph is a pair $H=(X, \mathcal{F})$, where $X=\left\{x_{1}, \ldots, x_{n}\right\}$ is a finite set (called vertex set of $H$ ) and $\mathcal{F}=\left\{Y_{1}, \ldots, Y_{m}\right\}$ is a non-empty family of subsets $Y_{1}, \ldots, Y_{m}$ of $X$. The elements $x_{1}, \ldots, x_{n}$ are called vertices of $H$ and the subsets $Y_{1}, \ldots, Y_{m}$ of $X$ are called hyperedges of $H$. An hypergraph on $X$ is a hypergraph having $X$ as vertex set.

Particular classes of hypergraphs can be introduced as follows

- If all the hyperedges of $H$ have the same cardinality $k$ we say that $H$ is a $k$-uniform hypergraph.

- If $H$ is a $k$-uniform hypergraph whose hyperedges are distinct, we say that $H$ is a simple $k$-uniform hypergraph.

- In particular, when $X=\hat{n}, 0 \leq k \leq n$ and $\mathcal{F}=\left(\begin{array}{l}\hat{n} \\ k\end{array}\right)$, we call the hypergraph $H:=\left(\hat{n},\left(\begin{array}{c}\hat{n} \\ k\end{array}\right)\right)$ the complete $(n, k)$ uniform hypergraph and (with abuse of notation) we denote it simply by $\left(\begin{array}{c}\hat{n} \\ k\end{array}\right)$. 
Remark 2.15. In this definition, it is required that $X$ and $\mathcal{F}$ are non-empty but not that all vertices belong to a hyperedge, nor that all hyperedges must have elements. Some authors give a more stringent definition by considering that there cannot exist isolated vertices, that is $\bigcup_{i}^{m} Y_{i}=X$ [5]. On the other hand, more general definitions are also introduced, by permitting also that $X$ or $\mathcal{F}$ can be empty [9]. In this last case the hypergraph with $X=\mathcal{F}=\emptyset$ is named empty and the one with $X \neq \emptyset$ and $\mathcal{F}=\emptyset$ is named trivial. Finally, let us note that if some $Y_{i}=\{x\}$ then the vertex $x$ is not isolated.

Remark 2.16. Let us notice that a simple $k$-uniform hypergraph $H=(X, \mathcal{F})$ can be always identified with a sub-hypergraph of $\left(\begin{array}{c}\hat{n} \\ k\end{array}\right)$, where $n=|X|$. This is done by identifying $x_{i}$ with $i$, for $i=1, \ldots, n$.

\section{Hypergraphs as Information Systems and Formal Contexts}

In this section, we show that there exists a bijection between hypergraphs and formal contexts/Boolean information systems. This bijection is constructively given in Proposition 3.4, after having showed how to obtain a Boolean information table from a hypergraph. It is also showed that (general) information tables can be put in relation with $k$-uniform hypergraphs. Then, we construct the concept lattice arising from a hypergraph.

\subsection{Equivalence results}

Let us start by defining an information table from a hypergraph.

Definition 3.1. Let $H$ be a hypergraph on a set $X=\left\{x_{1}, \ldots, x_{n}\right\}$, with hyperedges $Y_{1}, \ldots, Y_{m}$. We associate to $H$ a Boolean Information System $\Gamma(H)$ defined as follows. The attribute set of $\Gamma(H)$ is $X$ and the object set of $\Gamma(H)$ is $\left\{Y_{1}, \ldots, Y_{m}\right\}$. The value set $V(\Gamma)$ is $\{0,1\}$ and the information map $F_{H}$ is defined naturally as

$$
F_{H}\left(Y_{i}, x_{j}\right):= \begin{cases}1 & \text { if } x_{j} \in Y_{i} \\ 0 & \text { otherwise }\end{cases}
$$

Example 3.2. Let $H$ be the hypergraph with vertex set $X=\{1,2,3,4\}$ and hyperedges $Y_{1}=\emptyset, Y_{2}=\{1\}, Y_{3}=\{2,3\}, Y_{4}=\{2,3\}, Y_{5}=\{1,2,4\}$. Then the information table $T[\Gamma(H)]$ is the following:

\begin{tabular}{|l|l|l|l|l|}
\hline & 1 & 2 & 3 & 4 \\
\hline$\emptyset$ & 0 & 0 & 0 & 0 \\
\hline$\{1\}$ & 1 & 0 & 0 & 0 \\
\hline$\{2,3\}$ & 0 & 1 & 1 & 0 \\
\hline$\{2,3\}$ & 0 & 1 & 1 & 0 \\
\hline$\{1,2,4\}$ & 1 & 1 & 0 & 1 \\
\hline
\end{tabular}

Definition 3.3. Let $\mathcal{J}=\langle U, A t t, V, F\rangle$ be an information system. We say that $\mathcal{J}$ is a Hypergraphic Information System if there exists a hypergraph $H$ such that $\mathcal{J} \cong \Gamma(H)$. 
In the next proposition we describe the indiscernibility relation in a hypergraphic information system.

Proposition 3.4. Let $H$ be a hypergraph on a set $X=\left\{x_{1}, \ldots, x_{n}\right\}$, with hyperedge family $\mathcal{F}=\left\{Y_{1}, \ldots, Y_{m}\right\}$ and $\Gamma(H)=<\mathcal{F}, X,\{0,1\}, F_{H}>$ the associated Boolean information system. Let $A \subseteq X$. Then, if $I_{A}$ is the A-indiscernibility relation in $\Gamma(H)$, we have that

$$
Y_{i} I_{A} Y_{j} \Longleftrightarrow Y_{i} \cap A=Y_{j} \cap A
$$

for all $i, j \in\{1, \ldots, m\}$.

Let us note that, by Proposition 3.4, if $Y$ is a hyperedge of $H$ then

$$
[Y]_{A}=\left\{Y_{i} \in \mathcal{F}: Y_{i} \cap A=Y \cap A\right\} .
$$

As simple corollaries of the above theorem, we have the following results on the A-degree of dependency of a set of attributes and the precision of a partial implication.

Corollary 3.5. With the same notations of Proposition 3.4, if $y$ is a subfamily of $\mathcal{F}$, then

$$
\mu_{y}^{A}(Y)=\frac{\left|\left\{Y_{i} \in \mathcal{y}: Y_{i} \cap A=Y \cap A\right\}\right|}{\left|\left\{Y_{i} \in \mathcal{F}: Y_{i} \cap A=Y \cap A\right\}\right|}
$$

for all $Y \in \mathcal{F}$.

Proof. The identity (7) follows directly from (6) and from the general definition of rough membership function given in (2).

Corollary 3.6. With the same notations of Proposition 3.4, if $A, B \subseteq X$ we have that

$$
\gamma_{A}(B)=\frac{\left|\left\{Y_{i} \in \mathcal{F}:\left(Y \in \mathcal{F} \wedge Y \cap A=Y_{i} \cap A\right) \Longrightarrow Y \cap B=Y_{i} \cap B\right\}\right|}{m} .
$$

Proof. It is easy to verify that $\operatorname{Pos}_{A}(B)=\left\{Y_{i} \in \mathcal{F}:\left[Y_{i}\right]_{A} \subseteq\left[Y_{i}\right]_{B}\right\}$, therefore the thesis follows directly by (6) and the general definition of $A$-degree dependence given in (3).

The result established in the next proposition tell us that the notion of a Boolean information table (and hence also of a formal context) is exactly equivalent to the notion of hypergraphic information system.

Proposition 3.7. Let $\mathcal{J}=\langle U, A t t, V, F\rangle$ be an information system. Then $\mathcal{J}$ is hypergraphic if and only if $\mathcal{J}$ is Boolean.

Moreover, if $\mathcal{J}=\langle U, A t t,\{0,1\}, F\rangle$ is a Boolean information table, then the hypergraph $H$ such that $\Gamma(H)$ is isomorphic to $\mathcal{J}$, has as vertex set the family $X=$ Att and as hyperedges the family $\mathcal{F}=\left\{Y_{u}: u \in U\right\}$ where each $Y_{u}=\{a \in A t t: F(u, a)=1\}$, under the convention of assuming $Y_{u} \neq Y_{u^{\prime}}$ for any arbitrary pair $u \neq u^{\prime}$.

Proof. An easy consequence of the definitions.

Example 3.8. Let us consider the formal context (or Boolean information system), given in table 3 .

It is equivalent to the hypergraph $(X, \mathcal{F})$ with $X=\{$ Furniture, Computers, Copy Machines, Specialized Machines $\}$ and $\mathcal{F}=\{\{\mathrm{F}, \mathrm{C}, \mathrm{Cm}, \mathrm{Sm}\},\{\mathrm{F}, \mathrm{C}\},\{\mathrm{C}, \mathrm{Cm}, \mathrm{Sm}\},\{\mathrm{C}\},\{\mathrm{C}, \mathrm{Cm}, \mathrm{Sm}\}$ ' $\}$. Let us remark that the objects Instruction and Contracts give rise to two equal hyperedges, which are however considered distinct: $\{\mathrm{C}, \mathrm{Cm}, \mathrm{Sm}\}$ and $\{\mathrm{C}, \mathrm{Cm}, \mathrm{Sm}\}$ '. 


\begin{tabular}{|c||c|c|c|c|}
\hline & Furniture & Computers & Copy Machines & Specialized Machines \\
\hline Consulting & 1 & 1 & 1 & 1 \\
\hline Planning & 1 & 1 & 0 & 0 \\
\hline Instruction & 0 & 1 & 1 & 1 \\
\hline Training & 0 & 1 & 0 & 0 \\
\hline Contracts & 0 & 1 & 1 & 0 \\
\hline
\end{tabular}

Table 3: Concept table taken from page 25 of [17].

Remark 3.9. We notice that the construction of a hypergraph from a formal context given in [15] is different. Indeed, in [15] the hyperedges are only the set of attributes (maximal with respect to set inclusion) corresponding to single objects: $\left\{\{g\}^{\uparrow} \subseteq M: g \in\right.$ $G,\{g\}^{\uparrow}$ is maximal with respect to set inclusion $\}$. In particular, in this way, a simple hypergraph is always obtained.

Moreover, if the Boolean information table is the result of a nominal scaling, we have the following result.

Corollary 3.10. If $\mathcal{J}$ is the Boolean information table corresponding to the nominal scaling of a many-valued context (aka an Information System), then the hypergraph $H$ such that $\Gamma(H)$ is isomorphic to J is $k$-uniform, with $k=|A t t|$.

Proof. A simple application of Proposition 2.12.

Example 3.11. Let us consider the information system in Table 5.1. The vertex set of the corresponding hypergraph is $X=\{\mathrm{H}-\mathrm{yes}, \mathrm{H}-\mathrm{no}, \mathrm{M}-\mathrm{yes}, \mathrm{M}-\mathrm{no}, \mathrm{T}-\mathrm{n}, \mathrm{T}-\mathrm{h}, \mathrm{T}-\mathrm{vh}, \mathrm{F}-\mathrm{y}$, F-n $\}$ and the hyperedges are $\mathcal{F}=\{\{$ H-no, M-yes, T-h, F-y $\},\{$ H-yes, M-no, T-h, F-n $\}$, $\{$ H-yes, M-yes, T-vh, F-y $\},\{$ H-no, M-yes, T-n, F-n $\},\{$ H-yes, M-no, T-h,F-n $\},\{$ H-yes, M-yes, T-n, F-y $\}$. As expected each hyperedge has four elements, one for each attribute of the original information table.

In Section 4 , we will study in detail the sub-class of complete $k$-uniform hypergraphs. In terms of Boolean information tables, they correspond to the case where for any possible combination of $k$ 1s among the attributes, there exists one object that exactly has these combinations. Thus, it is impossible that a Boolean information table generated through nominal scaling is complete, since given an attribute just only one value can be set to one. Of course with other scaling techniques, results could be different.

The results of this subsection are summarized in the schema of Figure 1 where "scaled I.T." indicates the class of hypergraphs obtainable by the nominal scaling of an information table.

\subsection{The Concept Lattice of Hypergraphs}

As already shown in Section 2, Boolean information table and formal contexts are two equivalent ways to represent data. So, by the previous Proposition 3.7, one deduces that formal context and hypergraph are also equivalent. Therefore, a formal context denoted by $\Psi(H)=(G, M, \mathcal{R})$ can be associated to a hypergraph $H=(X, \mathcal{F})$, where 


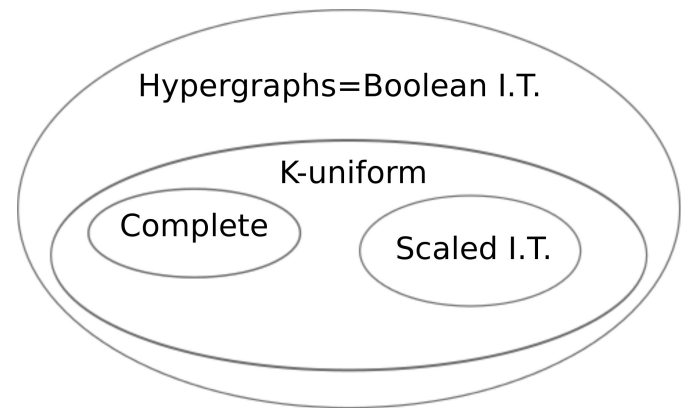

Figure 1: Relationship among hypergraphs and information tables.

$G:=\mathcal{F}=\left\{Y_{1}, \ldots, Y_{m}\right\}, M:=X=\left\{x_{1}, \ldots, x_{n}\right\}$ and the relation $\mathcal{R} \subseteq \mathcal{F} \times X$ is defined naturally as

$$
Y_{i} \mathcal{R} x_{j}: \Longleftrightarrow x_{j} \in Y_{i}
$$

The notion of formal concept can thus be interpreted in the case of the formal context $\Psi(H)$ induced by an hypergraph $H=(X, \mathcal{F})$ as follows. Let $\mathcal{O} \subseteq \mathcal{F}$ and $A \subseteq X$ be respectively a subset of objects and a subset of attributes of $\Psi(H)$. We set $O:=\bigcap\{Y$ : $Y \in \mathcal{O}\}$ and $\mathcal{A}:=\{Y \in \mathcal{F}: A \subseteq Y\}$. Then:

(i) $\mathcal{O}$ is the extent of some formal concept of $\Psi(H)$ if and only if $\mathcal{O}=\{Y \in \mathcal{F}: O \subseteq Y\}$. In this case, $\mathcal{O}$ is the extent of the formal concept $(\mathcal{O}, O)$.

(ii) $A$ is the intent of some formal concept of $\Psi(H)$ if and only if $A=\bigcap\{Y: Y \in \mathcal{A}\}=$ $\bigcap\{Y \in \mathcal{F}: A \subseteq Y\}$. In this case, $A$ is the intent of the formal concept $(\mathcal{A}, A)$.

Moreover, $\mathcal{O}^{\uparrow}=\bigcap\{Y: Y \in \mathcal{O}\}$ and $A^{\downarrow}=\{Y \in \mathcal{F}: A \subseteq Y\}$.

As a consequence of these facts, we can compute the precision (confidence) of a partial attribute implication as given in Definition 2.10.

Corollary 3.12. Let $H=(X, \mathcal{F})$ be a hypergraph. Let $A$ and $B$ be two sets of attributes of $\Psi(H)$. Then

$$
\delta_{A}(B)=\frac{|\{Y \in \mathcal{F}: A \cup B \subseteq Y\}|}{|\{Y \in \mathcal{F}: A \subseteq Y\}|}
$$

and $\delta_{A}(B)=1$ if $\{Y \in \mathcal{F}: A \subseteq Y\}=\emptyset$.

Proof. The statement follows directly by (4) and the above facts.

Let us remark that we have assumed that if $\mathcal{A}$ is the empty family, then $\bigcap\{Y: Y \in$ $\mathcal{A}\}=X$.

\section{The Hypergraphic System $\left.\Gamma\left(\begin{array}{c}\hat{n} \\ k\end{array}\right)\right)$}

In this section we apply the general results established in the previous sections on a well known hypergraph model, that is the complete $(n, k)$ uniform hypergraph $\left(\begin{array}{c}\hat{n} \\ k\end{array}\right)$ given in definition 2.14, for $n \geq k$ fixed. Through a combinatorial study of the structure of 
the hypergraphic information system $\left.\Gamma\left(\begin{array}{l}\hat{n} \\ k\end{array}\right)\right)$ we will then be able to answer questions (1) and (2) of example 1.1.

At first, let us give an example of $(\mathrm{n}, \mathrm{k})$ uniform hypergraph and equivalent information system.

Remark 4.1. In several numerical examples we will deal with set of sets. In order to simplify the notation, we will use the string notation $x_{1} \ldots x_{k}$ instead of the set notation $\left\{x_{1}, \ldots, x_{k}\right\}$. For example, 1234 will denote the set $\{1,2,3,4\}$.

Example 4.2. Let us consider the information system $\mathcal{J}=\Gamma\left(\left(\begin{array}{l}\hat{4} \\ 3\end{array}\right)\right)$, whose information table is the following:

\begin{tabular}{|l|l|l|l|l|}
\hline & 1 & 2 & 3 & 4 \\
\hline$\{1,2,3\}$ & 1 & 1 & 1 & 0 \\
\hline$\{1,2,4\}$ & 1 & 1 & 0 & 1 \\
\hline$\{1,3,4\}$ & 1 & 0 & 1 & 1 \\
\hline$\{2,3,4\}$ & 0 & 1 & 1 & 1 \\
\hline
\end{tabular}

Let $A=\{1,2\}$. Then

$$
\pi_{A}(\mathcal{J})=\{\{123,124\},\{134\},\{234\}\} .
$$

So that

$\mathbb{C O}_{A}(\mathcal{J})=\{\emptyset,\{134\},\{234\},\{123,124\},\{134,234\},\{123,124,134\},\{123,124,234\},\{123,124,134,234\}\}$,

whose diagram is the following:

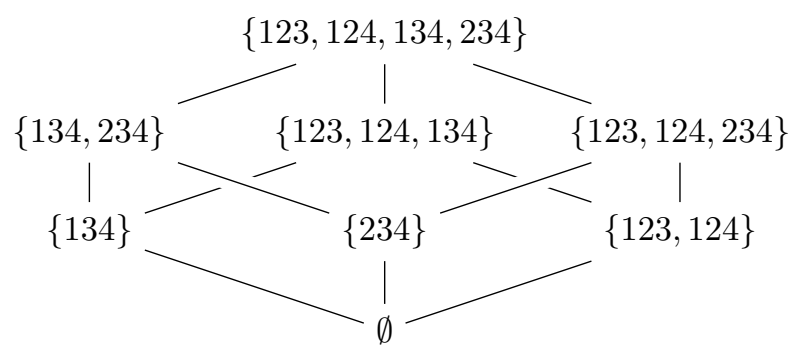

Hence, according to remark 2.2 , the Boolean algebra $\mathbb{C O}_{A}(\mathcal{J})$ is isomorphic to $\mathcal{P}(\hat{3})$.

By using Proposition 3.4 we obtain the following useful characterization of the $A$ indiscernibility classes in $\left.\Gamma\left(\begin{array}{l}\hat{n} \\ k\end{array}\right)\right)$, where $A$ is a subset of $\hat{n}$.

Proposition 4.3. Let $\mathcal{J}$ be the Boolean information system $\left.\Gamma\left(\begin{array}{c}\hat{n} \\ k\end{array}\right)\right)$ where $n \geq k$ are positive integer and let $A \subseteq \hat{n}$. Then, there exists a bijection between the set $\pi_{A}(\mathcal{J})$ of all $A$-indiscernibility classes in $\Gamma(H)$ and the set

$$
\Sigma_{A}:=\{S \subseteq A: \max \{0, k+a-n\} \leq|S| \leq \min \{a, k\}\},
$$

where $a:=|A|$. 
Proof. Let $S \in \Sigma_{A}$ and set $s:=|S|$. By Proposition 3.4 all $k$-subsets of $\hat{n}$ whose intersection with $A$ is equal to $S$, if non empty, is an $A$-indiscernibility class in J. But if $\max \{0, k+a-n\} \leq s \leq \min \{a, k\}$ then there exists $\left(\begin{array}{c}n-a \\ k-s\end{array}\right) k$-subsets of $\hat{n}$ such that their intersection with $A$ is $S$. On the other hand if $x \in\left(\begin{array}{c}\hat{n} \\ k\end{array}\right)$, then the cardinality $s$ of $S:=x \cap A$ satisfies $\max \{0, k+a-n\} \leq s \leq \min \{a, k\}$. In fact $s \geq 0, s \leq a$ and $s \leq k$ by definition of $S$. Moreover it holds that $S=x \cap A=x \backslash(\hat{n} \backslash A)$ and so $s \geq k-(n-a)=k+a-n$. We proved that the function that associates to each $S \in \Sigma_{A}$ the $A$-indiscernibility class $C:=\left\{x \in\left(\begin{array}{c}\hat{n} \\ k\end{array}\right): x \cap A=S\right\}$ is bijective with inverse that associates to each $C \in \pi_{A}(\mathcal{J})$ the subset $S:=A \cap\left(\bigcap_{x \in C} x\right)$.

Corollary 4.4. Let J be the Boolean information system $\left.\Gamma\left(\begin{array}{l}\hat{n} \\ k\end{array}\right)\right)$ where $n \geq k$ are positive integers and let $A \subseteq \hat{n}$ and $x \in\left(\begin{array}{c}\hat{n} \\ k\end{array}\right)$. By setting $a:=|A|, S:=x \cap A$ and $s:=|S|$ we have $\max \{0, k+a-n\} \leq s \leq \min \{a, k\}$ and $\left|[x]_{A}\right|=\left(\begin{array}{c}n-a \\ k-s\end{array}\right)$.

Proof. As we have seen in the first part of the proof of Proposition 4.3 the $A$-indiscernibility class $[x]_{A}$ is the set of all $k$-subsets of $\hat{n}$ whose intersection with $A$ is equal to $S$, $\max \{0, k+a-n\} \leq s \leq \min \{a, k\}$ and $\left|[x]_{A}\right|=\left(\begin{array}{c}n-a \\ k-s\end{array}\right)$.

Remark 4.5. Let $H=(\hat{n}, \mathcal{F})$ be a simple $k$-uniform hypergraph on $n$ vertices (i.e. $\mathcal{F}$ is a sub-family of $\left.\left(\begin{array}{l}\hat{n} \\ k\end{array}\right)\right)$. Let $\mathcal{J}:=\Gamma(H)$ and $A$ a subset of the attribute set $\hat{n}$.

In order to compute the number of distinct equivalence classes in the set $\pi_{A}(\mathcal{J})$ and the complete list of all the objects of any indiscernibility class $[x]_{A} \in \pi_{A}(\mathcal{J})$ it is convenient to see $\mathcal{J}$ as a binary matrix $T(\mathcal{J})$. To the attribute set $A$ we can associate in a natural way a $\left(\begin{array}{l}n \\ k\end{array}\right) \times|A|$ submatrix $T_{A}(\mathcal{J})$ of $T(\mathcal{J})$ by choosing the columns corresponding to the elements of A. By using standard topological sort algorithms we can easily find the number of distinct rows of $T_{A}(\mathcal{J})$ and partition the rows of $T_{A}(\mathcal{J})$ with respect to the equality relation.

In the following result we determine the number of elements in any indiscernibility partition $\pi_{A}(\mathcal{J})$ when $\mathcal{J}=\Gamma\left(\left(\begin{array}{c}\hat{n} \\ k\end{array}\right)\right)$.

Proposition 4.6. Let $\mathcal{J}=\Gamma\left(\left(\begin{array}{l}\hat{n} \\ k\end{array}\right)\right)$. If $A$ is a subset of attributes of $\mathcal{J}$ such that $|A|=l$, then:

(i) if $l \leq k$ we have $\left|\pi_{A}(\mathcal{J})\right|=\sum_{i=0}^{\min \{l, n-k\}}\left(\begin{array}{c}l \\ l-i\end{array}\right)$;

(ii) if $l>k$ we have $\left|\pi_{A}(\mathcal{J})\right|=\sum_{i=0}^{\min \{k, n-l\}}\left(\begin{array}{c}l \\ k-i\end{array}\right)$.

Proof. Without loss of generality we can assume that $A=\{1,2, \ldots, l\}$. By Proposition 3.4 we have that, given two $k$-subsets $S_{1}, S_{2}$ of $[n], S_{1} I_{A} S_{2}$ if and only if $S_{1} \cap A=S_{2} \cap A$. Then we can associate to each class in $\pi_{A}(\mathcal{J})$ a subset of $A$. In particular for each subset $B$ of $A$ we can consider the $k$-subsets $S$ of $[n]$ such that $S \cap A=B$. This set is either empty or it is an equivalence class in $\pi_{A}(\mathcal{J})$. Such a class has $\left(\begin{array}{c}n-l \\ k-|B|\end{array}\right)$ elements that can be obtained by choosing $k-|B|$ elements in $[n] \backslash A$.

Let us suppose now $l \leq k$. The $k$-subsets of $[n]$ containing $A$ form a class in $\pi_{A}(\mathcal{J})$. More generally however we fix a subset $B$ of $A$, the set of the $k$-subsets of $[n]$ containing $B$, if it is not empty, it matches an equivalence class of $I_{A}$ with $\left(\begin{array}{c}n-l \\ k-|B|\end{array}\right)$ elements. Such a set is empty if and only if $k-|B| \leq n-l$ which is equivalent to $l-|B| \leq n-k$. Thus it follows that the number of classes in $\pi_{A}(\mathcal{J})$ is equal to

$$
\left|\pi_{A}(\mathcal{J})\right|=\sum_{j=0}^{n-k}\left(\begin{array}{l}
l \\
j
\end{array}\right)=\sum_{i=0}^{n-k}\left(\begin{array}{c}
l \\
l-i
\end{array}\right)
$$


and thus (i) holds.

If $l>k$ then obviously for each subset $B$ of $A$ such that $|B|>k$ there exists no $k$-subset $S$ of $[n]$ such that $S \cap A=B$. When $|B|=k$ the unique $k$-subset $S$ of $[n]$ containing $B$ is $B$. So each $k$-subset in $A$ uniquely identifies a class in $\pi_{A}(\mathcal{J})$. As before if $|B|<k$ and $k-|B| \leq n-l$ then the equivalence class of $I_{A}$ of all the $k$-subsets $S$ such that $S \cap A=B$ contains $\left(\begin{array}{c}n-l \\ k-|B|\end{array}\right)$ elements. Finally if $|B|<k$ and $k-|B|>n-l$ then there is not an element $S \in\left(\begin{array}{c}{[n]} \\ k\end{array}\right)$ such that $S \cap A=B$. Then when $l>k$ the number of classes in $\pi_{A}(\mathcal{J})$ is thus equal to

$$
\left|\pi_{A}(\mathcal{J})\right|=\sum_{j=l-n+k}^{k}\left(\begin{array}{l}
l \\
j
\end{array}\right)=\sum_{i=0}^{n-l}\left(\begin{array}{c}
l \\
k-i
\end{array}\right)
$$

and the proposition is proved.

By virtue of the result established in Proposition 4.6 it is convenient to set

$$
c(n, l, k):= \begin{cases}\sum_{i=0}^{\min \{l, n-k\}}\left(\begin{array}{c}
l \\
l-i
\end{array}\right) & \text { if } l \leq k \\
\sum_{i=0}^{\min \{k, n-l\}}\left(\begin{array}{c}
l \\
k-i
\end{array}\right) & \text { if } l>k\end{cases}
$$

when $n, l$ and $k$ are three integers such that $0 \leq l, k \leq n$. Clearly, $c(n, l, k)$ is the answer to question 1 in example 1.1, whereas the answer to question 2 is given by the procedure outlined in Remark 4.5.

We obtain then the following result concerning $\mathbb{C O}_{A}\left(\left(\begin{array}{l}\hat{n} \\ k\end{array}\right)\right)$.

Corollary 4.7. If $A$ is a subset of attributes of $\left.\Gamma\left(\begin{array}{l}\hat{n} \\ k\end{array}\right)\right)$ such that $|A|=l$ then $\left.\mathbb{C O} \mathbb{O}_{A}\left(\Gamma\left(\begin{array}{l}\hat{n} \\ k\end{array}\right)\right)\right)$ is a Boolean algebra isomorphic to $\left\langle\mathcal{P}(\hat{s}), \subseteq, \cap, \cup,{ }^{c}, \emptyset, \hat{s}\right\rangle$, where $s=c(n, l, k)$.

Proof. The thesis is a direct consequence of Proposition 4.7, Proposition 4.6 and of (10).

In the next proposition we determine a general formula of the dependency degree between two attribute subsets of the information system $\left.\Gamma\left(\begin{array}{c}\hat{n} \\ k\end{array}\right)\right)$.

Proposition 4.8. Let $\left.\mathcal{J}=\Gamma\left(\begin{array}{l}\hat{n} \\ k\end{array}\right)\right)$ and let $A, B$ be two attribute subsets of $\mathcal{J}$ (i.e. $A, B \subseteq$ $A t t=\hat{n})$ such that $a=|A|$ and $b=|B|$. Then

$$
\gamma_{A}(B)=\left\{\begin{array}{cc}
1 & \text { if } B \subseteq A \\
\left(\left(\begin{array}{l}
a \\
k
\end{array}\right)+\left(\begin{array}{c}
a \\
k+a-n
\end{array}\right)\right)\left(\begin{array}{l}
n \\
k
\end{array}\right)^{-1} & \text { otherwise. }
\end{array}\right.
$$

Proof. By definition of $A$-degree dependency $\gamma_{A}(B)$ is equal to the sum of the cardinality of the indiscernibility classes in $\pi_{A}(\mathcal{J})$ that are contained in some classes in $\pi_{B}(\mathcal{J})$. Let $A_{j} \in \pi_{A}(\mathcal{J})$. By Proposition $4.3 A_{j}$ is the subset of $\left(\begin{array}{l}\hat{n} \\ k\end{array}\right)$ whose intersection with $A$ is the subset

$$
S=A \cap\left(\bigcap_{x \in A_{j}} x\right),
$$


and $s:=|S|$ satisfies $\max \{0, k+a-n\} \leq|S| \leq \min \{a, k\}$. In this case $A_{j}=\left\{x \in\left(\begin{array}{l}\hat{n} \\ k\end{array}\right)\right.$ : $x \cap A=S\}$.

Let us suppose first that $B \subseteq A$. Set $T:=S \cap B \subseteq A$ and $t:=|T|$. By definition of $T$ we have $t \geq 0, t \leq b$ and $t \leq s \leq k$. Moreover since $S \cup B \subseteq A$ it holds

$$
t=s+b-|S \cup B| \geq s+b-a \geq k+a-n+b-a=k+b-n .
$$

Thus by Proposition 4.3 again to $T$ we can associate an indiscernibility class $B_{i} \in \pi_{B}(\mathcal{J})$ such that $B_{i}=\left\{x \in\left(\begin{array}{l}\hat{n} \\ k\end{array}\right): x \cap B=T\right\}$. If $x \in A_{j}$ then $x \cap B=x \cap A \cap B=S \cap B=T$, so $A_{j} \subseteq B_{i}$. Then each class in $\pi_{A}(\mathcal{J})$ is contained in a suitable class in $\pi_{B}(\mathcal{J})$ and then $\gamma_{A}(B)=1$.

Let us suppose now $B \backslash A \neq \emptyset$. If $s=k$ or $s=k+a-n$ then $A_{j}$ contains only a $k$-element subset of $\hat{n}$ and trivially it is contained in some $B_{i} \in \pi_{B}(\mathcal{J})$. Let now $\max \{0, k+a-n+1\} \leq|S| \leq \min \{a, k-1\}$ and let $p \in B \backslash A$. In this case there exist two $k$-subsets $x_{1}, x_{2} \in A_{j}$ such that $p \in x_{1} \backslash x_{2}$. It follows that $x_{1} \cap B \neq x_{2} \cap B$ and thus by Proposition $3.4 x_{1}$ is not equivalent to $x_{2}$ with respect to $I_{B}$. Thus $A_{j}$ is not contained in any class in $\pi_{B}(\mathcal{J})$. Therefore the only indiscernibility classes in $\pi_{A}$ which are in some $l_{A}(B)$ are the set of $k$-subsets of $\hat{n}$ whose intersection $S$ with $A$ has cardinality $s=k$ or $s=k+a-n$, and in both cases such classes are singletons.

We determine now the core and the reducts of the hypergraphic system $\Gamma\left(\left(\begin{array}{l}\hat{n} \\ k\end{array}\right)\right)$.

Proposition 4.9. Let $n, k$ be two positive integers with $k<n$ and let $\mathcal{J}=\Gamma\left(\left(\begin{array}{l}\hat{n} \\ k\end{array}\right)\right)$. Then $\operatorname{CORE}(\mathcal{J})=\emptyset$ and $\operatorname{RED}(\mathcal{J})=\left(\begin{array}{c}\hat{n} \\ n-1\end{array}\right)$.

Proof. Let $A$ be a $(n-1)$-subset of $\hat{n}$. By Proposition 4.6, we have $\left|\pi_{A}(\mathcal{J})\right|=\sum_{i=0}^{1}\left(\begin{array}{c}n-1 \\ k-i\end{array}\right)=$ $\left(\begin{array}{c}n-1 \\ k\end{array}\right)+\left(\begin{array}{c}n-1 \\ k-1\end{array}\right)=\left(\begin{array}{l}n \\ k\end{array}\right)=\left|\pi_{A t t}(\mathcal{J})\right|$. Since $\left|\pi_{A}(\mathcal{J})\right| \leq\left|\pi_{A t t}(\mathcal{J})\right|$, we obtain $\pi_{A}(\mathcal{J})=\pi_{A t t}(\mathcal{J})$. Let now $B \in\left(\begin{array}{c}\hat{n} \\ n-2\end{array}\right)$ and let $S$ be a subset of $B$ with $|S|=k-1$. Then there exist two subsets of $\hat{n}$ whose intersection with $B$ is exactly $S$, namely $K=S \cup\{u\}$ and $K^{\prime}=S \cup\left\{u^{\prime}\right\}$, where $u, u^{\prime} \in \hat{n} \backslash B$ with $u \neq u^{\prime}$. Thus by Proposition $3.4, K I_{B} K^{\prime}$ and thus $\pi_{B}(\mathcal{J}) \neq \pi_{A}(\mathcal{J})$. It follows that each $(n-1)$-subset of $\hat{n}$ is a reduct of $\mathcal{J}$. Their intersection, which is equal to $C O R E(\mathcal{J})$ by Proposition 2.5, is empty.

Example 4.10. Let us consider the family of information systems $\Gamma\left(\left(\begin{array}{l}\hat{5} \\ k\end{array}\right)\right)$ for $k<5$. The set of reducts is the same for all the information systems of this family and it is:

$$
R E D\left(\Gamma\left(\left(\begin{array}{l}
\hat{5} \\
k
\end{array}\right)\right)\right)=\left(\begin{array}{l}
\hat{5} \\
4
\end{array}\right)=\{1234,1245,1345,1235,2345\} .
$$

In proposition 4.6 we have studied the hypergraph $\left(\begin{array}{l}\hat{n} \\ k\end{array}\right)$ from the point of view of a Boolean information system. We examine now the hypergraph $\left(\begin{array}{l}\hat{n} \\ k\end{array}\right)$ from the point of view of a formal context. At first we study the concept lattice of the formal context $\Psi\left(\left(\begin{array}{l}\hat{n} \\ k\end{array}\right)\right)$.

Example 4.11. Let us consider the hypergraph $\left(\begin{array}{l}\hat{4} \\ 2\end{array}\right)$. By section 3.2 we obtain immediately the following concept lattice of the formal context $\Psi\left(\left(\begin{array}{l}\hat{4} \\ 2\end{array}\right)\right)$. The diagram of this lattice is the following: 


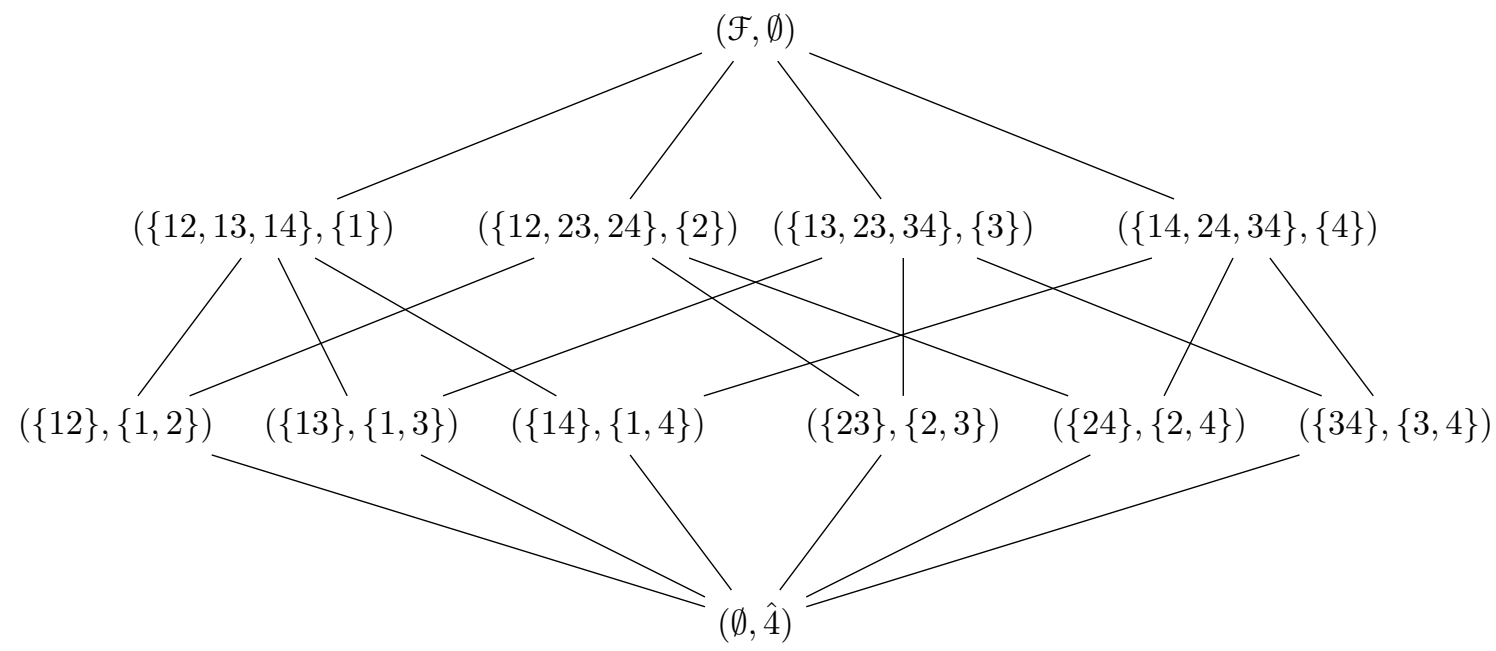

In order to describe the general form of the concept lattice of $\Psi\left(\left(\begin{array}{l}\hat{n} \\ k\end{array}\right)\right)$, we set

$$
\mathcal{V}(n, k):=\{K \subseteq \hat{n}:|K| \leq k\} \cup\{\hat{n}\} .
$$

$(\mathcal{V}(n, k), \subseteq)$ is obviously a sub-poset of the Boolean algebra $(\mathcal{P}(\hat{n}), \subseteq)$. We have the following result:

Proposition 4.12. Let $1 \leq k<n$ and $\mathbb{K}:=\Psi\left(\left(\begin{array}{c}\hat{n} \\ k\end{array}\right)\right)$. Then:

(i) The set of the formal concepts of $\mathbb{K}$ is: $\mathfrak{B}(\mathbb{K})=\left\{\left(A^{\uparrow}, A\right): A \in \mathcal{V}(n, k)\right\}$, where $A^{\uparrow}=\left\{Y \in\left(\begin{array}{c}\hat{n} \\ k\end{array}\right): A \subseteq Y\right\}$.

(ii) The concept lattice $(\mathfrak{B}(\mathbb{K}), \sqsubseteq)$ is isomorphic to the dual poset of $(\mathcal{V}(n, k), \subseteq)$.

Proof. By section 3.2, $A$ is the intent of the formal concept $\left(A^{\uparrow}, A\right)$ if and only if $A=$ $\bigcap\left\{Y \in\left(\begin{array}{c}\hat{n} \\ k\end{array}\right): A \subseteq Y\right\}$. Thus if $A$ is the intent of some formal concept either $|A| \leq k$ or $A=\hat{n}$. On the other hand, since $k<n$, if $A$ is a subset of $\hat{n}$ of cardinality less than or equal to $k$ then, for each $j \in \hat{n} \backslash A$, there exists always a $k$-subset $K$ of $\hat{n}$ containing $A$ but not containing $j$. Thus, again by section $3.2, A$ is the intent of some formal concept. Moreover, $A=\hat{n}$ is the intent of the formal concept $(\emptyset, \hat{n})$. Thus $(i)$ holds.

To prove (ii) let us consider the map $\phi: \mathfrak{B}(\mathbb{K}) \rightarrow \mathcal{V}(n, k)$ given by $\phi\left(A^{\uparrow}, A\right):=A$. By the previous part it follows that $\phi$ is bijective. It remains to prove that it is orderreversing. This follows directly from the definition of the order relations.

Remark 4.13. Notice that if $k=n$ then $\mathcal{V}(n, k)$ is the power set of $\hat{n}$, while by section 3.2 we see that there is the formal concept $\left.\left(\begin{array}{l}\hat{n} \\ k\end{array}\right), \hat{n}\right)$ in $\mathfrak{B}(\mathbb{K})$. So Proposition 4.12 does not hold in this case.

For the formal context $\left.\Psi\left(\begin{array}{l}\hat{n} \\ k\end{array}\right)\right)$ we can establish a result analogous to Proposition 4.8. In other terms, we can exactly determine the precision of the implication between two any attribute subsets of $\Psi\left(\left(\begin{array}{c}\hat{n} \\ k\end{array}\right)\right)$. 
Proposition 4.14. Let $A$ and $B$ be two attribute subsets of $\Psi\left(\left(\begin{array}{l}\hat{n} \\ k\end{array}\right)\right)$. Then

$$
\delta_{A}(B)=\left\{\begin{array}{cl}
\left(\begin{array}{c}
n-|A \cup B| \\
k-|A \cup B|
\end{array}\right) /\left(\begin{array}{l}
n-|A| \\
k-|A|
\end{array}\right) & \text { if }|A| \leq k \\
1 & \text { otherwise. }
\end{array}\right.
$$

Proof. By Corollary 3.12 we have that

$$
\delta_{A}(B)=\frac{\left|\left\{Y \in\left(\begin{array}{l}
\hat{n} \\
k
\end{array}\right): A \cup B \subseteq Y\right\}\right|}{\left|\left\{Y \in\left(\begin{array}{l}
\hat{n} \\
k
\end{array}\right): A \subseteq Y\right\}\right|}
$$

and $\delta_{A}(B)=1$ if $\left\{Y \in\left(\begin{array}{l}\hat{n} \\ k\end{array}\right): A \subseteq Y\right\}=\emptyset$. Now, if $|A| \leq k$ then $\left\{Y \in\left(\begin{array}{c}\hat{n} \\ k\end{array}\right): A \subseteq Y\right\} \neq \emptyset$ and we have that $\left|\left\{Y \in\left(\begin{array}{l}\hat{n} \\ k\end{array}\right): A \subseteq Y\right\}\right|=\left(\begin{array}{l}n-|A| \\ k-|A|\end{array}\right),\left|\left\{Y \in\left(\begin{array}{l}\hat{n} \\ k\end{array}\right): A \cup B \subseteq Y\right\}\right|=\left(\begin{array}{l}n-|A \cup B| \\ k-|A \cup B|\end{array}\right)$, hence the first identity in (13) follows directly by (14). Finally, if $|A|>k$ then $\{Y \in$ $\left.\left(\begin{array}{l}\hat{n} \\ k\end{array}\right): A \subseteq Y\right\}=\emptyset$ and this provides the second identity in (13).

It would be interesting to extend all the results of this section to more general Boolean information tables. In particular, a formula to compute the attribute dependence (propositions 4.8 and 4.14 ) would be welcome for any generic Boolean information table. As an approximation one could try to use the results of these propositions to give a lower/upper bound for other Boolean information systems. Let us note however that if we take a generic Boolean information table without any delimiting condition, a trivial bound can only be given.

\section{The Granular Partition Lattice of Hypergraphs}

In this section we study the collection of all partitions generated by a hypergraphic system.

In general, let $X=\left\{x_{1}, \ldots, x_{n}\right\}$ be a finite set and denote by $\pi(X)$ or simply by $\pi$ a partition of $X$ in $M$ disjoint blocks $B_{i} \subseteq X$. We write $\pi(X):=\left\{B_{1}, \ldots, B_{M}\right\}$ to denote such a partition and $\Pi(X)$ to denote the set of all the partitions of $X$. It is well known that a partial order on the set $\Pi(X)$ can be given as follows:

for all $\pi, \pi^{\prime} \in \Pi(X)$

$$
\pi \preceq \pi^{\prime}: \Longleftrightarrow(\forall B \in \pi)\left(\exists B^{\prime} \in \pi^{\prime}\right): B \subseteq B^{\prime}
$$

The set $\Pi(X)$ with the partial order $\preceq$ is a lattice, and it is called partition lattice of the set $X$. We denote by $\hat{0}_{\Pi(X)}$ the so-called discrete partition $\left\{\left\{x_{1}\right\},\left\{x_{2}\right\}, \ldots,\left\{x_{n}\right\}\right\}$, and by $\hat{1}_{\Pi(X)}$ the so-called trivial partition $\{X\}$, therefore respectively the minimum and the maximum of the lattice $\Pi(X)$. See [11], [7] for a discussion about these lattice properties.

Now, let $\mathcal{J}=\langle U, A t t, V, F\rangle$ be a given information system, where $U=\left\{u_{1}, \ldots, u_{n}\right\}$. Once fixed a set of attributes $A \subseteq A t t$, through the $A$-indiscernibility relation we obtain the partition of the universe $\pi_{A}(\mathcal{J})=\left\{[u]_{A}: u \in U\right\}$. We set now

$$
\Upsilon(\mathcal{J}):=\left\{\pi_{A}(\mathcal{J}): A \subseteq A t t\right\} .
$$


Since $\Upsilon(\mathcal{J})$ is a subset of $\Pi(U)$, we can consider on $\Upsilon(\mathcal{J})$ the induced partial order $\preceq$ from $\Pi(U)$. Therefore the pair $(\Upsilon(\mathcal{J}), \preceq)$ is a sub-poset of the partition lattice $(\Pi(U), \preceq)$. The poset $(\Upsilon(\mathcal{J}), \preceq)$ is an important structure in granular computing and in rough set theory (see [35]) as well as in database theory (see [22]). Specifically, according to Yao (see [35]) the order structure $(\Upsilon(\mathcal{J}) \preceq)$ "can be used to develop a partition model of databases". Actually it is known (see $[22])$ that $(\Upsilon(\mathcal{J}), \preceq$ ) has a lattice structure. We denote, respectively, by $\hat{0}_{\mathcal{J}}$ and $\hat{1}_{\mathcal{J}}$ the minimum and the maximum of the lattice $(\Upsilon(\mathcal{J}), \preceq)$. Let us note here that $\hat{1}_{\mathcal{J}}$ always coincides with the maximum $\hat{1}_{\Pi(U)}=\{U\}$ of the partition lattice $\Pi(U)$, whereas, in general, $\hat{0}_{\mathcal{J}}$ may differ from the minimum $\hat{0}_{\Pi(U)}=\left\{\left\{u_{1}\right\},\left\{u_{2}\right\}, \ldots,\left\{u_{n}\right\}\right\}$ of $\Pi(U)$. Finally, we also recall that although $(\Upsilon(\mathcal{J}), \preceq)$ has a lattice structure with respect to the partial order $\preceq$ induced by $\Pi(U)$, it is not (in general) a sub-lattice of $(\Pi(U), \preceq)$. In fact, in [22] it was proved that while the meet of $(\Upsilon(\mathcal{J}), \preceq)$ coincides with the meet of $(\Pi(U), \preceq)$, their joins can be different. More precisely, the join on $\Upsilon(\mathcal{J})$ can be characterized in terms of the Galois connection that will be introduced in the next subsection.

Example 5.1. Let us consider the following information table J:

\begin{tabular}{|c||c|c|c|c|c|}
\hline $\mathcal{J}$ & $a_{1}$ & $a_{2}$ & $a_{3}$ & $a_{4}$ & $a_{5}$ \\
\hline$p_{1}$ & 0 & 1 & 0 & 1 & 0 \\
$p_{2}$ & 1 & 0 & 0 & 0 & 1 \\
$p_{3}$ & 1 & 1 & 1 & 1 & 0 \\
$p_{4}$ & 0 & 1 & 2 & 0 & 1 \\
$p_{5}$ & 1 & 0 & 0 & 0 & 1 \\
$p_{6}$ & 1 & 1 & 2 & 1 & 0 \\
\hline
\end{tabular}
have

Let us take the two attribute subsets $A=\left\{a_{3}\right\}$ and $B=\left\{a_{1}, a_{2}, a_{4}, a_{5}\right\}$. So that we

$$
\pi_{A}(\mathcal{J})=\left\{\left\{p_{1}, p_{2}, p_{5}\right\},\left\{p_{3}\right\},\left\{p_{4}, p_{6}\right\}\right\}, \pi_{B}(\mathcal{J})=\left\{\left\{p_{1}\right\},\left\{p_{2}, p_{5}\right\},\left\{p_{3}, p_{6}\right\},\left\{p_{4}\right\}\right\} .
$$

Then the join of $\pi_{A}(\mathcal{J})$ and $\pi_{B}(\mathcal{J})$ in $\Pi(U)$ is $\pi=\left\{\left\{p_{1}, p_{2}, p_{5}\right\},\left\{p_{3}, p_{4}, p_{6}\right\}\right\}$. However, $\pi$ is not an element of $\Upsilon(\mathcal{J})$ because there does not exist a set of attributes that generate it. On the other hand, the join of $\pi_{A}(\mathcal{J})$ and $\pi_{B}(\mathcal{J})$ in $\Upsilon(\mathcal{J})$ is $\pi_{\emptyset}(\mathcal{J})=\left\{\left\{p_{1}, p_{2}, p_{3}, p_{4}, p_{5}, p_{6}\right\}\right\}$.

In literature there is not a commonly used name in order to designate the lattice $(\Upsilon(\mathcal{J}), \preceq)$. In this paper we call it granular partition lattice:

Definition 5.2. Let $\mathcal{J}=\langle U, A t t, V, F\rangle$ be an information system. We call granular partition lattice of $\mathcal{J}$ the lattice $\left(\Upsilon(\mathcal{J}), \preceq, \hat{0}_{\mathcal{J}}, \hat{1}_{\mathcal{J}}\right)$, where $\hat{0}_{\mathcal{J}}=\pi_{\text {Att }}(\mathcal{J})$ and $\hat{1}_{\mathcal{J}}=\pi_{\emptyset}(\mathcal{J})=$ $\hat{1}_{\Pi(U)}$. In particular, if $H=(X, \mathcal{F})$ is a hypergraph, we call granular partition lattice of $H$, denoted by $\Upsilon(H)$, the granular partition lattice of $\Gamma(H)$.

We determine now the granular partition lattice of $\left(\begin{array}{l}\hat{n} \\ k\end{array}\right)$ for all $n \geq k \geq 1$.

Theorem 5.3. The granular partition lattice $\left(\Upsilon\left(\left(\begin{array}{l}\hat{n} \\ k\end{array}\right)\right), \preceq\right)$ is isomorphic to the dual lattice of $(\mathcal{V}(n, n-2), \subseteq)$ (see notation in equation $(12))$. 
Proof. As we proved in the proof of the Propostion 4.9 it holds that if $A \subseteq \hat{n}$ has cardinality $|A|>n-2$, then $\pi_{A}=\pi_{\hat{n}}$, while if $|A| \leq n-2$, then $\pi_{A} \neq \pi_{\hat{n}}$. Consider now the function $\phi: \Upsilon\left(\left(\begin{array}{l}\hat{n} \\ k\end{array}\right)\right) \longrightarrow \mathcal{V}(n, n-2)$ defined by:

$$
\phi\left(\pi_{A}\right)=\left\{\begin{array}{lll}
A & \text { if } & |A| \leq n-2, \\
\hat{n} & \text { if } & |A|>n-2 .
\end{array}\right.
$$

Let us prove now that $\phi$ is well defined. For this we have to prove that, if $\pi_{A}=\pi_{B}$ are two subsets of $\hat{n}$, then $\phi\left(\pi_{A}\right)=\phi\left(\pi_{B}\right)$. Let $A, B \subseteq \hat{n}$, with $|A| \leq n-2, B \leq n-2$ and $\phi\left(\pi_{A}\right)=A \neq B=\phi\left(\pi_{B}\right)$. Since $A \neq B$, we have $A \triangle B=(A \backslash B) \cup(B \backslash A) \neq \emptyset$. Without loss of generality we can assume $A \backslash B \neq \emptyset$. Let $a \in A \backslash B$ and let $K$ be a $k$-subset of $\hat{n}$ such that $a \in K$ and $|B \cap K|>|B|+k-n$. Note that such a $K$ exists in this case. Set $S:=B \cap K$ and let $K^{\prime} \in\left(\begin{array}{l}n \\ k\end{array}\right)$ such that $B \cap K^{\prime}=S$ and $a \notin K^{\prime}$. By Proposition 3.4, $K I_{B} K^{\prime}$ but $\neg\left(K I_{A} K^{\prime}\right)$ and thus $\pi_{A} \neq \pi_{B}$.

The map $\phi$ is clearly both injective and surjective. Let us prove it is an isomorphism. For this let $A, B \subset \hat{n}$ such that $\pi_{A} \preceq \pi_{B}$. If $|A| \geq n-1$, then $\phi\left(\pi_{A}\right)=\hat{n} \supseteq \phi\left(\pi_{B}\right)$, for each $B$. Let us suppose $|A|<n-1$. In this case, since $\pi_{A} \preceq \pi_{B}$, we have $|B|<n-1$. Thus $\phi\left(\pi_{A}\right)=A$ and $\phi\left(\pi_{B}\right)=B$. Since $\pi_{A} \neq \pi_{\hat{n}}$, there exist $K_{1}, K_{2} \in\left(\begin{array}{l}\hat{n} \\ k\end{array}\right)$ such that $K_{1} \neq K_{2}$ By Proposition 4.3 it holds $k+a-n<\left|K_{1} \cup A\right|=\left|K_{2} \cup A\right|<k$, where $a=|A|$. Let $b \in B \backslash A$. Then there exist $K_{1}^{\prime}, K_{2}^{\prime} \in\left(\begin{array}{c}\hat{n} \\ k\end{array}\right)$ such that $\left|K_{1} \cup A\right|=\left|K_{2} \cup A\right|=\left|K_{1}^{\prime} \cup A\right|=$ $\left|K_{2}^{\prime} \cup A\right|$ and $b \in K_{1}^{\prime} \backslash K_{2}^{\prime}$. This implies $K_{1}^{\prime} I_{A} K_{2}^{\prime}$ but $\neg\left(K_{1}^{\prime} I_{B} K_{2}^{\prime}\right)$, and this contradicts the condition $\pi_{A} \preceq \pi_{B}$. Thus $B \subseteq A$ and the theorem is proved.

\subsection{Indiscernibility Partition Lattice and Pattern Structures}

We establish now an interesting connection between the granular partition lattice and another order structure based on the notion of pattern structure as introduced in [16]. A pattern structure can be considered a generalization of the formal context notion. For more details concerning these structures we refer the reader to [16].

Definition 5.4. [16] $A$ pattern structure is a triple $\mathcal{S}=(G,(D, \sqcap), \delta)$, where $G$ is a set whose elements are called objects, $(D, \sqsubseteq)$ is a meet-semilattice whose inf-operation is denoted by $\sqcap$ and $\delta: G \rightarrow D$ is a function called description map. The elements of $D$ are called patterns. If $g \in G$, the pattern $\delta(g)$ is called the description of the object $g$ in $(D, \sqsubseteq)$.

If $\mathcal{S}=(G,(D, \sqcap), \delta)$ is a pattern structure, one can consider the following two square operators:

$$
A \in \mathcal{P}(G) \mapsto A^{\square}:=\prod_{g \in A} \delta(g) \in D
$$

and

$$
d \in D \mapsto d^{\square}:=\{g \in G: d \sqsubseteq \delta(g)\} \in \mathcal{P}(G)
$$

These operators form a Galois connection between the partially ordered sets $(\mathcal{P}(G), \subseteq)$ and $(D, \sqsubseteq)$.

If $A \in \mathcal{P}(G)$ and $d \in D$, the closures of $A$ and $d$ in $\mathcal{S}$ are respectively

$$
c l_{\mathcal{S}}(A):=\left(A^{\square}\right)^{\square} \text { and } c l_{\mathcal{S}}(d):=\left(d^{\square}\right)^{\square}
$$


Definition 5.5. [16] Let $\mathcal{S}=(G,(D, \sqcap), \delta)$ be a pattern structure. A pattern concept of $\mathcal{S}$ is an ordered pair $(A, d) \in \mathcal{P}(G) \times D$ such that $A^{\square}=d$ and $d^{\square}=A$.

We denote by $P C O(\mathcal{S})$ the set of all pattern concepts of $\mathcal{S}$. In [16] is considered the following partial order $\leqslant$ on $P C O(\mathcal{S})$. If $\left(A_{1}, d_{1}\right)$ and $\left(A_{2}, d_{2}\right)$ are two pattern concepts of $\mathcal{S}$ then

$$
\left(A_{1}, d_{1}\right) \leqslant\left(A_{2}, d_{2}\right): \Longleftrightarrow A_{1} \subseteq A_{2}\left(\Longleftrightarrow d_{2} \sqsubseteq d_{1}\right)
$$

It results then (see [16]) that the partially ordered set

$$
\mathbb{P}_{c o}(\mathcal{S}):=(P C O(\mathcal{S}), \leqslant)
$$

is a complete lattice, which is called pattern concept lattice of the pattern structure $\mathcal{S}$.

Now we recall a useful construction which associates a pattern structure to any information system in a very natural way. This construction was introduced in [3], where more details can be found.

In what follows, let $\mathcal{J}=\langle U, A t t, F, V a l\rangle$ be an information system.

Definition 5.6. [3] We denote by $\mathcal{J}_{p s}$ the pattern structure $(G,(D, \sqsubseteq), \delta)$ such that $G:=$ Att $,(D, \sqsubseteq):=(\Pi(U), \preceq)$ and $\delta(a):=\pi_{\{a\}}(\mathcal{J})$ for all $a \in$ Att. The pattern structure $\mathcal{J}_{p s}$ is called partition pattern structure of $\mathcal{J}$.

Remark 5.7. By Definition 5.6, if $A \subseteq A t t$ and $\pi \in \Pi(U)$, from (17) and (18) we obtain

$$
A^{\square}=\bigwedge_{a \in A} \pi_{\{a\}}(\mathcal{J})=\pi_{A}(\mathcal{J})
$$

and

$$
\pi^{\square}=\left\{a \in A t t: \pi \preceq \pi_{\{a\}}(\mathcal{J})\right\} .
$$

At this point we can prove the following isomorphism between the granular partition lattice of $\mathcal{J}$ and the pattern concept lattice of $\mathcal{J}_{p s}$.

Theorem 5.8. The granular partition lattice $(\Upsilon(\mathcal{J}), \preceq)$ is order-isomorphic to the dual lattice of $\mathbb{P}_{c o}\left(\mathcal{J}_{p s}\right)$.

Proof. By (20) and (21), the map $\phi: P C O\left(\mathcal{J}_{p s}\right) \longrightarrow \Upsilon(\mathcal{J})$ defined by $\phi(A, \pi):=\pi$, it is a lattice homomorphism. It is clearly injective because, if $\pi_{1}=\phi\left(A_{1}, \pi_{1}\right)=\phi\left(A_{2}, \pi_{2}\right)=\pi_{2}$, then $A_{1}=\pi_{1}^{\square}=\pi_{2}^{\square}=A_{2}$ and thus $\left(A_{1}, \pi_{1}\right)=\left(A_{2}, \pi_{2}\right)$. Moreover, by (21) it follows that the image of $\phi$ is contained in $\Upsilon(\mathcal{J})$.

Let now $\pi=\pi_{B}(\mathcal{J}) \in \Upsilon(\mathcal{J})$ and let $A:=\pi^{\square}$. Let us prove that $\pi_{A}(\mathcal{J})=\pi$. By $(21)$ and (22) we deduce that

$$
\pi \preceq \bigwedge_{a \in A} \pi_{\{a\}}(\mathcal{J})=\pi_{A}(\mathcal{J}) .
$$

On the other hand, let $b \in B$. Since $\{b\} \subseteq B$, we have $\pi=\pi_{B}(\mathcal{J}) \preceq \pi_{\{b\}}(\mathcal{J})$, so that $B \subseteq A$, and therefore $\pi_{A}(\mathcal{J}) \preceq \pi_{B}(\mathcal{J})=\pi$. Hence $\pi_{A}(\mathcal{J})=\pi$. This implies that $(A, \pi)$ is a pattern concept of $\mathcal{J}_{p s}$ and finally $\pi=\phi(A, \pi)$ is in the image of the map $\phi$. Thus the image of $\phi$ is equal to $\Upsilon(\mathcal{J})$ and the thesis follows.

By the previous theorem we obtain the following useful consequence. 
Corollary 5.9. Let $A \subseteq$ Att. Then $\pi_{A}(\mathcal{J})=\pi_{c l_{J_{p s}}(A)}(\mathcal{J})$.

Proof. By (19) and (21) we have

$$
\operatorname{cl}_{\mathcal{J}_{p s}}(A)=\left(A^{\square}\right)^{\square}=\pi_{A}(\mathcal{J})^{\square}
$$

On the other hand, by Theorem 5.8 the couple $\left(\pi_{A}(\mathcal{J})^{\square}, \pi_{A}(\mathcal{J})\right)$ is a pattern concept in $\mathcal{J}_{p s}$, therefore by (23) and (21) we have

$$
\pi_{A}(\mathcal{J})=\left(\pi_{A}(\mathcal{J})^{\square}\right)^{\square}=c l_{\mathcal{J}_{p s}}(A)^{\square}=\pi_{c l_{J_{p s}}(A)}(\mathcal{J}) .
$$

By means of the closure notion for the partition pattern structure $\mathcal{J}_{p s}$ introduced in Definition 5.6, we are also able to provide a link between the join of two indiscernibility partitions in $\Upsilon(\mathcal{J})$ and their corresponding join in $(\Pi(U), \preceq)$.

Proposition 5.10. Let $A, B \subseteq$ Att and let $\bar{A}:=\operatorname{cl}_{\mathcal{J}_{p s}}(A), \bar{B}:=c l_{\mathcal{J}_{p s}}(A)$. Then

$$
\left(\pi_{A} \vee \pi_{B}\right)_{\Upsilon(\mathcal{J})}=\pi_{\bar{A} \cap \bar{B}}(\mathcal{J})=c l_{\mathcal{J}_{p s}}\left(\left(\pi_{A} \vee \pi_{B}\right)_{\Pi(U)}\right),
$$

where $\left(\pi_{A} \vee \pi_{B}\right)_{\Upsilon(\mathcal{J})}$ and $\left(\pi_{A} \vee \pi_{B}\right)_{\Pi(U)}$ are the joins of $\pi_{A}(\mathcal{J})$ and $\pi_{B}(\mathcal{J})$, respectively in $\Upsilon(\mathcal{J})$ and in $(\Pi(U), \preceq)$.

Proof. Let $\bar{A}:=\operatorname{cl}_{\mathcal{J}_{p s}}(A)$ and $\bar{B}:=c l_{\mathcal{J}_{p s}}(B)$, so that $\bar{A}=\left(\pi_{A}\right)^{\square}$ and $\bar{B}=\left(\pi_{B}\right)^{\square}$ by (21). On the other hand, by (22) we also have

$$
\begin{aligned}
{\left[\left(\pi_{A} \vee \pi_{B}\right)_{\Pi(U)}\right]^{\square} } & =\left\{a \in A t t:\left(\pi_{A} \vee \pi_{B}\right)_{\Pi(U)} \preceq \pi_{a}(\mathcal{J})\right\} \\
& =\left\{a \in A t t: \pi_{A}(\mathcal{J}) \preceq \pi_{\{a\}}(\mathcal{J}) \text { and } \pi_{B}(\mathcal{J}) \preceq \pi_{\{a\}}(\mathcal{J})\right\}=\bar{A} \cap \bar{B} .
\end{aligned}
$$

Then, by (24) and (21) we deduce that

$$
c l_{\mathcal{J}_{p s}}\left(\left(\pi_{A} \vee \pi_{B}\right)_{\Pi(U)}\right)=(\bar{A} \cap \bar{B})^{\square}=\pi_{\bar{A} \cap \bar{B}}(\mathcal{J}) .
$$

Let us note now that $\pi_{\bar{A} \cap \bar{B}}(\mathcal{J})$ is an upper bound of $\pi_{A}(\mathcal{J})$ and $\pi_{B}(\mathcal{J})$ in $\Upsilon(\mathcal{J})$. In fact, by Corollary 5.9 we have $\pi_{A}(\mathcal{J})=\pi_{\bar{A}}(\mathcal{J}) \preceq \pi_{\bar{A} \cap \bar{B}}(\mathcal{J})$, because $\bar{A} \cap \bar{B} \subseteq \bar{A}$. In a similar way we obtain $\pi_{B}(\mathcal{J}) \preceq \pi_{\bar{A} \cap \bar{B}}(\mathcal{J})$.

Let now $C \subseteq A t t$ such that $\pi_{A}(\mathcal{J}) \preceq \pi_{C}(\mathcal{J})$ and $\pi_{B}(\mathcal{J}) \preceq \pi_{C}(\mathcal{J})$. We set $\bar{C}:=c l_{\mathcal{J}_{p s}}(C)=$ $\left(\pi_{C}(\mathcal{J})\right)^{\square}$. By Corollary 5.9 we have $\pi_{\bar{A}}(\mathcal{J}) \preceq \pi_{\bar{C}}(\mathcal{J})$ and $\pi_{\bar{B}}(\mathcal{J}) \preceq \pi_{\bar{C}}(\mathcal{J})$. Then, since $\left(\bar{A}, \pi_{A}\right),\left(\bar{B}, \pi_{B}\right)$ and $\left(\bar{C}, \pi_{C}\right)$ are all pattern concepts in $\mathcal{J}_{p s}$, by (20) we obtain $\bar{C} \subseteq \bar{A}$ and $\bar{C} \subseteq \bar{B}$. Thus $\bar{C} \subseteq \bar{A} \cap \bar{B}$ and finally $\pi_{\bar{A} \cap \bar{B}}(\mathcal{J}) \preceq \pi_{\bar{C}}(\mathcal{J})=\pi_{C}(\mathcal{J})$. This shows that $\pi_{\bar{A} \cap \bar{B}}(\mathcal{J})$ is the join of $\pi_{A}(\mathcal{J})$ and $\pi_{B}(\mathcal{J})$ in $\Upsilon(\mathcal{J})$ and the thesis follows.

\section{Conclusion}

In this work, we presented a unified picture for bridging hypergraph theory with Formal Concept Analysis and Rough Set Theory. Results about formal equivalence between hypergraphs and formal context/Boolean information tables are surveyed. Further, we 
have seen that a sub-class of $k$-uniform hypergraphs corresponds to Boolean information tables obtained by a scaling procedure of many-valued ones. These equivalences are the starting point for using hypergraph methods in FCA and RST and vice versa. Here, we developed in detail the case of k-uniform complete hypergraphs and we were able, using combinatorial techniques, to study the indiscernibility classes, the functional dependencies and the reducts in the equivalent Boolean information systems.

For the future, we plan to extend our results to non-complete $k$-uniform hypergraphs in order to capture any information table through nominal scaling. Other techniques to scale from a many-valued to a Boolean situation could also be considered and the corresponding hypergraph investigated. The general idea is to develop new techniques to study FCA and RST using tools from hypergraph theory and vice versa, to investigate the typical FCA and RST instruments in hypergraphs. Examples are:

- connect the minimal transversal hypergraph with rough set reducts and use algorithms to solve one problem to study the other [12, 13];

- define a dynamical system on hypergraphs which represents the idea to move from information tables to their rough set reducts and then iteratively to the reducts of reducts and so on. It can be proved that the dynamical system has a fixed point which can characterize the original information table. Similarly, it is possible to define a dynamical system on hypergraphs representing the transition from a formal context to its intents and show that also this system reaches a fix point which summarizes the essential features of the original formal context.

Finally, due to the generality of formal contexts and information tables, many links to other disciplines can be find, for instance with Chu spaces and Scott systems [24, 34, 39]. All these connections are worth to investigate in order to build a whole picture and to make all discipline cross fertilize each other.

\section{References}

[1] P. Alexandroff, Diskrete Räume, Mathematiceskij Sbornik 2 (1937), 501-518.

[2] F.G. Arenas, Alexandroff spaces, Acta Math. Univ. Comenianae (1999), 17-25.

[3] J. Baixeries, M. Kaytoue, A. Napoli, Characterizing functional dependencies in formal concept analysis with pattern of Ann. Math. Artif. Intell. 72(1-2), 2014, pp. 129-149

[4] C. Berge, Graphs and Hypergraphs, North Holland P. C., Amsterdam-London, 1973.

[5] C. Berge, Hypergraphs: Combinatorics of Finite Sets, Elsevier, Amsterdam, 1984.

[6] G. Birkhoff, Lattice Theory, American Mathematical Society, Providence, Rhode Island, Third Edition, 1967

[7] D. Bianucci, G. Cattaneo, Information Entropy and Granulation Co-Entropy of Partitions and Coverings: a Summary, in: Special Issue on Foundations of Rough Sets, J.F. Peter and A. Skowron Eds., Transactions on Rough Sets X, Lecture Notes in Computer Science, vol. 5656, Springer-Verlag, 2009, pp. 15-66.

[8] B. Bollobás, Combinatorics: Set Systems, Hypergraphs, Families of Vectors, and Combinatorial Probability, Cambridge University Press, 1986.

[9] A. Bretto, Hypergraph Theory: An Introduction. Springer, 2013

[10] G. Cattaneo, An investigation about rough set theory: some foundational and mathematical aspects, Fundamenta Informaticae 108, 2011, pp. 197-221.

[11] G. Cattaneo, D. Ciucci, D. Bianucci, Entropy and Co-entropy of Partitions and Coverings with Applications to Roughness Theory, in: Granular Computing: At the Junction of Rough Sets and Fuzzy sets. Series: Studies on Fuzziness and Soft Computing. R. Bello, R. Falcon, W Pedrycz and J. Kacprzyk, Eds., vol. 224, Springer-Verlag, 2008, pp. 55-77. 
[12] Chiaselotti, G., Ciucci, D., Gentile, T.: Granular Geometry on Simple Graphs, submitted to Information Sciences, second revision (2015)

[13] Chiaselotti, G., Ciucci, D., Gentile, T., Infusino, F.: Generalizations of Rough Set Tools inspired by Graph Theory, Rough Set Theory Workshop, Warsaw (2015)

[14] G. Chen, N. Zhong, Y. Yao, A Hypergraph Model of Granular Computing, in: Proc. IEEE International Conference on Granular Computing, 2008, pp 130-135.

[15] P. Colomb, L. Nourine, About Keys of Formal Context and Conformal Hypergraph, in: Formal Concept Analysis, Lecture Notes in Computer Science, vol. 4933, 2008, pp. 140-149.

[16] B. Ganter, S. O. Kuznetsov, Pattern Structures and Their Projections, ICCS 2001, LNCS2120, 2001, 129-142

[17] B. Ganter, R. Wille, Formal Concept Analysis. Mathematical Foundations, Springer-Verlag, 1999.

[18] B. Ganter, S. O. Kuznetsov, Scale Coarsening as Feature Selection. In: R.Medina, S.Obiedkov, Eds., Proc. 6th International Conference on Formal Concept Analysis (ICFCA 2008), Lecture Notes in Artificial Intelligence, Vol. 4933, 2008, pp. 217-228

[19] B. Ganter, C. Meschke, A Formal Concept Analysis Approach to Rough Data Tables, T. Rough Sets 14, 2011, pp. 37-61

[20] M. Grötschel, L. Lovász, Combinatorial Optimization, in: Handbook of Combinatorics, Elsevier Science, 1995, 1541-1597.

[21] S.O. Kuznetsov, J. Poelmans, Knowledge representation and processing with formal concept analysis, Wiley Interdisc. Rew.: Data Mining and Knowledge Discovery 3(3), 2013, pp. 200-215

[22] T.T. Lee, An Information-Theoretic Analysis of Relational Databases - part I: Data Dependencies and Metric, IEEE Transactions on Software Engineering 13 (1987), pp. 1049-1061.

[23] M. Luxenburger, Implication partielles dans un contexte, Mathématiques et sciences humaines 113, 1991, 35-55

[24] P. Pagliani, Information quanta and approximation spaces. I. Non-classical approximation operators. GrC 2005, 605-610, 2005

[25] P. Pagliani, M.K. Chakraborty, Formal topology and Information Systems, Transactions on Rough Sets VI, Lecture Notes in Computer Science, vol. 4374, 2007, pp. 253-297

[26] Z. Pawlak, Rough sets - Theoretical aspects of reasoning about data, Kluwer Academic Publishers, Dordrecht, 1991

[27] Z. Pawlak, A. Skowron, Rudiments of rough sets, Information Sciences 177, 2007, pp. 3-27

[28] N. Pasquier, Y. Bastide, R. Taouil, L. Lakhal, Efficient Mining of Association Rules Using Closed Itemset Lattices, Information Systems 24(1), 1999, 25-46

[29] A. Skowron, C. Rauszer, The Discernibility Matrices and Functions in Information Systems, Intelligent Decision Support, Theory and Decision Library series , vol. 11, Springer Netherlands, 1992 pp. 331-362.

[30] J.G. Stell, Relational Granularity for Hypergraphs, in: Rough Sets and Current Trends in Computing, Lecture Notes in Computer Science, vol. 6086, Springer-Verlag, 2010, pp. 267-276.

[31] J.G. Stell, Formal Concept Analysis over Graphs and Hypergraphs, in Formal Concept Analysis, in: Graph Structures for Knowledge Representation and Reasoning, Lecture Notes in Computer Science, vol. 8323, 2014, pp. 165-179.

[32] P. Wasilewski, Concept lattices vs. approximation spaces, in: RSFDGrC 2005, Lecture Notes in Computer Science, vol. 3641, 2005, pp. 114-123

[33] M. Wolski, Formal concept analysis and rough set theory from the perspective of finite topological approximations, in: Transactions on Rough Sets III, Lecture Notes in Computer Science, vol. 3400, 2005, pp. 230-243

[34] M. Wolski, A. Gomolinska, Concept Formation: Rough Sets and Scott Systems. Fundamenta Informaticae, 127(1-4), 2013, pp. 17-33

[35] Y.Y. Yao, A Partition Model of Granular Computing, in: Transactions on Rough Sets I, Lecture Notes in Computer Science, vol. 3100, Springer-Verlag, 2004, pp. 232-253.

[36] Y.Y. Yao, A Comparative Study of Formal Concept Analysis and Rough Set Theory in Data Analysis, in: Rough Sets and Current Trends in Computing, Lecture Notes in Computer Science, vol. 3066, Springer-Verlag, 2004, pp. 59-68

[37] Y.Y. Yao, Concept lattices in rough set theory, in: Proc. NAFIPS'04. vol. 2, IEEE, 2004, pp. 796-801.

[38] Y.Y. Yao, Y.Chen, Rough Set Approximations in Formal Concept Analysis, in: Transactions on Rough Sets V, Lecture Notes in Computer Science, vol. 4100, Springer-Verlag, 2006, pp. 285-305.

[39] G.Q. Zhang, G. Shen, Approximable concepts, Chu spaces and information systems, Theory and Application of Categories, vol.17, 2006, pp. 80-102. 